sensitive Plant survey of Piute Valley TMA

BLM Lit Denver 


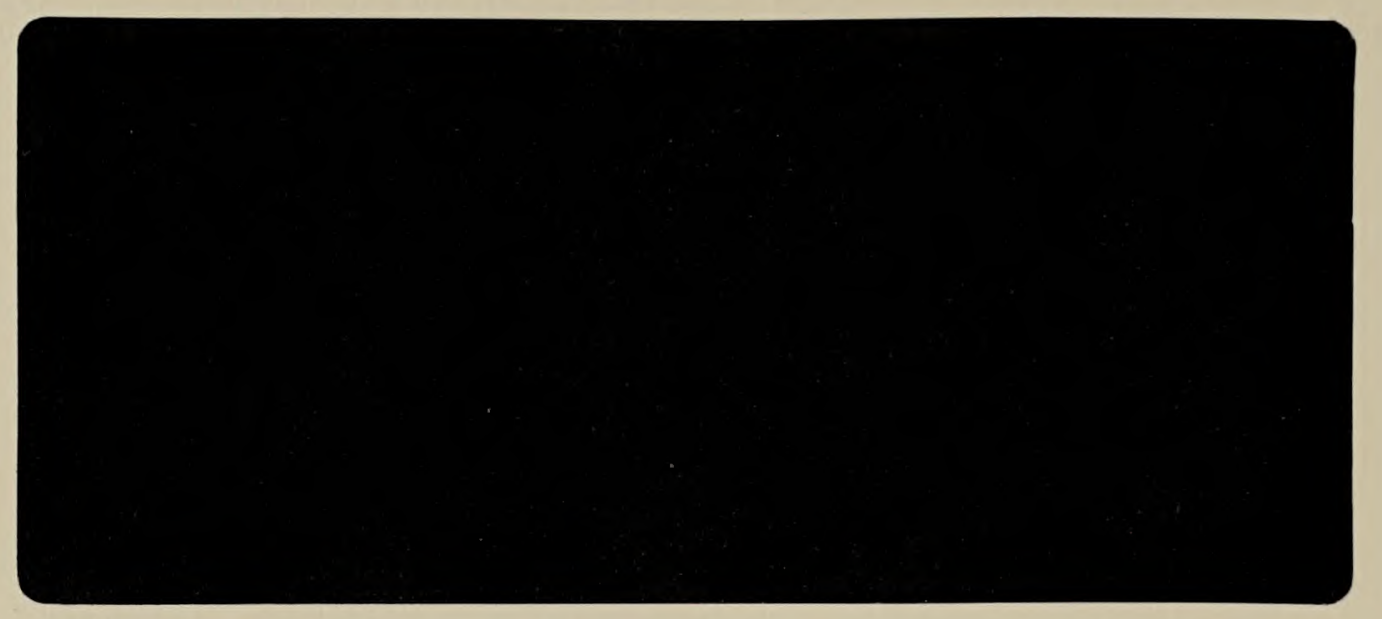




\section{Sensitive Plant Survey \\ of Piute Valley TMA}

BLM Library

Denver Federal Center Bldg. 50, OC-521

P.O. Box 25047

Denver, CO 80225

\section{Prepared by}

Gayle Marrs-Smith

Botanist, BLM, Las Vegas District 



\section{TABLE OF CONTENTS}

1 Vegetation . . . . . . . . . . . . . . . . . . 1

1.1 General Description . . . . . . . . . . . . . 1

1.2 Description of Vegetation Communities....... . . 1

2 Endangered, Threatened, or Sensitive Plants . . . . . . 4

3 References . . . . . . . . . . . . . . . . . . 14 APPENDIX • . . . . . . . . . . . . . . . . . 15 



\section{LIST OF TABLES AND FIGURES}

\section{Figures}

Figure 1. Vegetation communities of Piute Valley TMA. . . . 2

Figure 2. Sensitive plant occurrence in Piute Valley TMA. . 11

Figure 3. Distribution of Penstemon bicolor ssp. roseus . . 13

\section{Tables}

Table 1. Plant species observed in Piute Valley. Relative abundance is as follows: $C=$ common, widespread throughout community type(s) or a community dominant; 0 occasional, scattered throughout community type(s) or locally abundant; $\mathrm{R}=$ rare, few seen anywhere. Introduced taxa are shown by "*". . . . . . . . . . . . . .

Table 2. Endangered, Threatened, or Sensitive Plants reported from Piute Valley TMA. 



\section{Vegetation}

\subsection{General Description}

The Piute Valley TMA is composed mainly of Mojave Desert scrub typical of the region. The dominant shrub, creosotebush (Larrea tridentata), floristically links the plant communities in the Piute Valley TMA. The Mojave Desert indicator species, Joshua tree (Yucca brevifolia), occurs throughout most of Piute valley. The dominant plant community types are various associations of creosotebush, yucca (Yucca brevifolia, Y. baccata, Y. schidigera), white bursage (Ambrosia dumosa), little leaf ratany (Krameria parvifolia), and blackbrush (Coleogyne ramosissima). In addition, the flora of Piute Valley is influenced by the proximity of the Colorado River. Because of the river's lower elevation, some plants more typical of the sonoran desert occur, such as brittlebush (Encelia farinosa), and have become established in the surrounding environs.

The vegetation of the Piute Valley TMA ranges from moderately to highly disturbed. Only a few areas display an unbroken, welldeveloped desert pavement, the surface crust preventing soil erosion. Large areas of the valley are quite sandy with a noncemented surface. Other indicators of rangeland disturbance are the following introduced annual species which are abundant in spring in Piute Valley: red brome (Bromus rubens); six-weeks fescue (Vulpia octoflora); Schismus barbatus; and heron's bill (Erodium cicutarium). Past land uses that contributed to disturbance include (1) grazing, (2) off-highway vehicle (OHV) use, (3) mining, and (4) road clearing for transmission line and pipeline construction. Currently, Piute Valley TMA is closed to grazing, competitive OHV events, and casual off-road use (due to road closures), but is still open to mining, rights-of-way, and casual recreation.

\subsection{Description of Vegetation Communities}

The following description of the vegetation of the Piute Valley TMA is based on Cronquist et al. (1972), who summarized the vegetation structure of the Intermountain Region. Four communities are represented within the boundaries of the TMA: creosote-bursage scrub, mixed Mojave shrubland, blackbrush, and pinyon-juniper woodland, all of which intergrade with one another. Wash vegetation in Piute Valley for the most part does not vary greatly in composition from the surrounding vegetation, except in some of the larger washes (e.g. Piute Wash). Piute Valley does not have perennial water and thus has no true riparian community. A map of the vegetation communities in the Piute Valley TMA is presented in Figure 1 . 



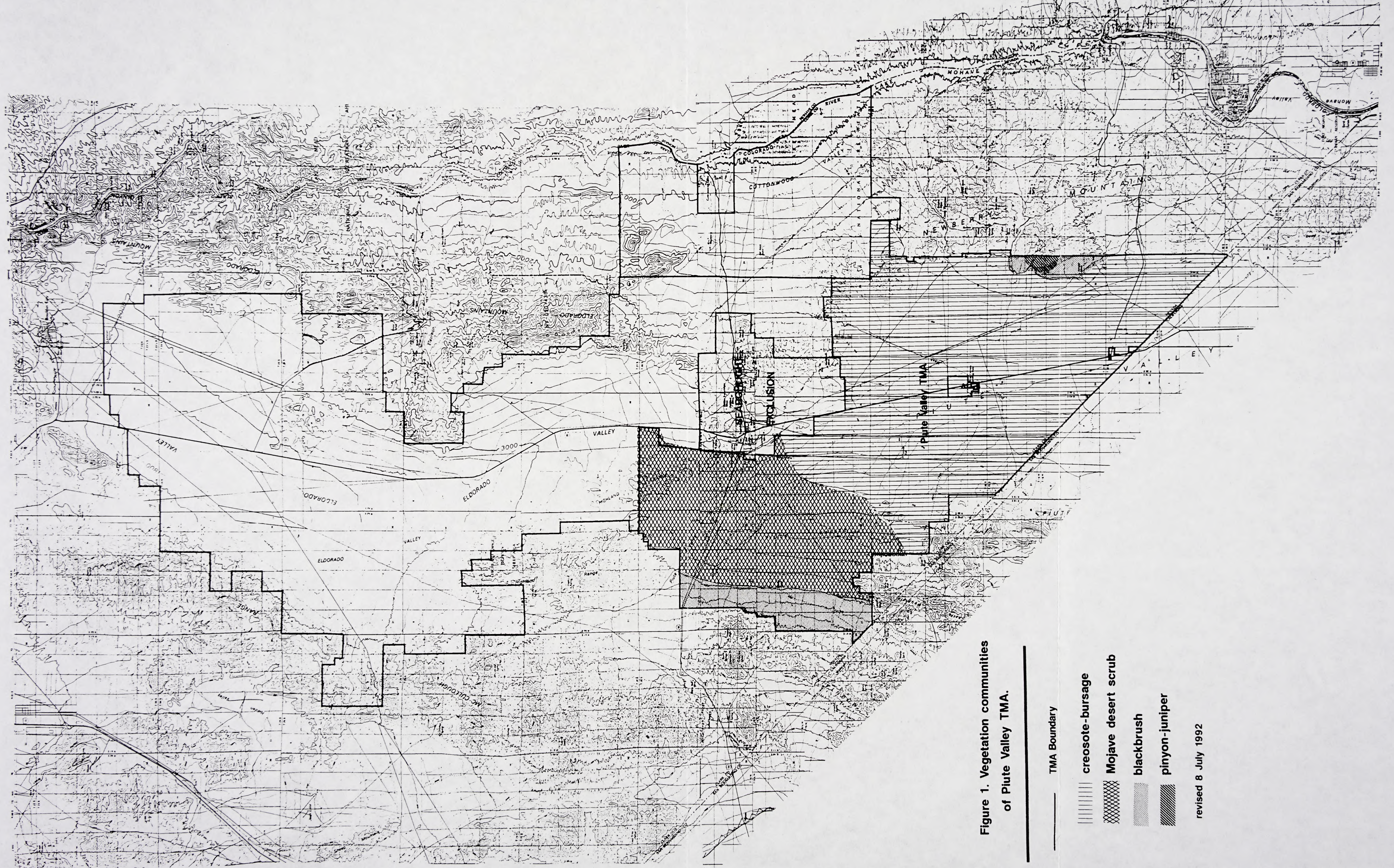



The creosote-bursage community occupies the sandy alluvial valley bottom and lower bajadas of Piute Valley, between 750 - $1000 \mathrm{~m}$ elevation. The overall plant diversity here is somewhat lower compared to the higher elevation areas surrounding the valley bottom. This community is represented by the co-dominants, creosotebush and bursage, with the following sub-dominants: little leaf ratany (Krameria parvifolia); cheesebush (Hymenoclea salsola), and goldenhead (Acamptopappus sphaerocephalus). The dominant grass, galleta (Hilaria rigida), forms robust swales within the creosote-bursage community.

The mixed Mojave desert shrub community occurs on upper bajadas and hills at elevations from about 1000 to $1250 \mathrm{~m}$. This community includes Joshua tree, Mohave yucca ( $Y$. schidigera), banana yucca ( $Y$. baccata), wolfberry (Lycium andersonii), Mormon-tea (Ephedra nevadensis), buckwheat (Eriogonum fasciculatum), sage (Salvia dorrii), horsebrush (Tetradymia spinescens), and Encelia virginensis. Spiny hopsage (Grayia spinosa) and winterfat (Ceratoides lanata) occur occasionally. In addition, this community includes a wide assemblage of cacti. The important grasses in this community are galleta, black grama (Bouteloua eriopoda), bush muhly (Muhlenbergia porteri), and needlegrass (Stipa speciosa).

The blackbrush scrub community, dominated by blackbrush (Coleogyne ramosissima), intergrades with the mixed Mojave desert shrub community on rocky, well-drained hillsides at elevations around $1250 \mathrm{~m}$ throughout the piute valley TMA. It also occurs at lower elevations on north-facing slopes of hills in the northern part of the Piute valley TMA (foothills of the Highland Range). The subdominants include Mohave yucca, Mormon tea, wolfberry, buckhorn cholla (Opuntia acanthocarpa), and barrel cactus (Ferocactus acanthodes).

Within the boundaries of the TMA, the pinyon-juniper woodland community occurs only at the highest elevations $(1250 \mathrm{~m})$ in the lower out-wash areas of the Newberry Mountains; here only Utah juniper (Juniperus osteosperma) is represented. Intergrading with Mojave desert shrub and blackbrush communities, the key species of this community include Nolina (Nolina bigelovii), green Mormon tea (Ephedra viridis), and Mojave sage (Salvia mohavensis).

As stated previously, wash vegetation in most of the Piute valley TMA does not significantly differ in species composition from the surrounding vegetation. Better drainage, occasional surface flows, and deep moisture availability enhance the wash vegetation, thus making washes lusher than adjacent areas. The exceptions are the larger washes in Piute Valley (e.g. Piute Wash), where distinct plant associations occur, dominated by desert willow (Chilopsis linearis), cheesebush, bladder sage (Salazaria mexicana), cat-claw (Acacia greggii), rabbitbrush (Chrysothamnus spp.), Porophyllum gracile, Ambrosia eriocentra, and desert almond (Prunus 


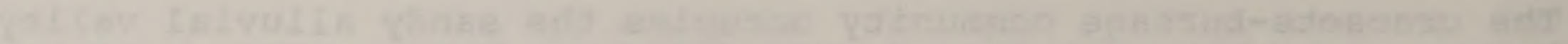

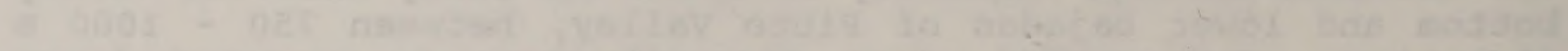

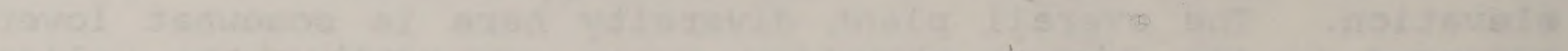

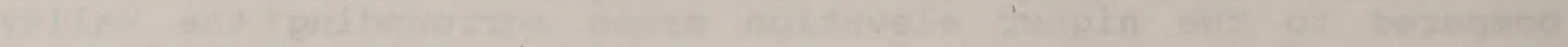

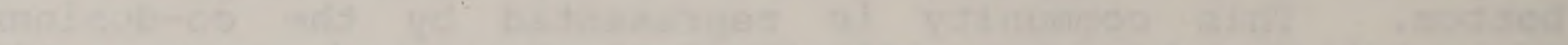

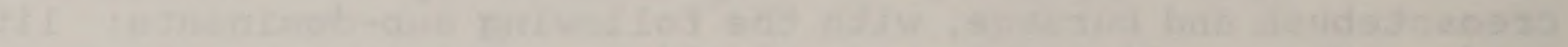

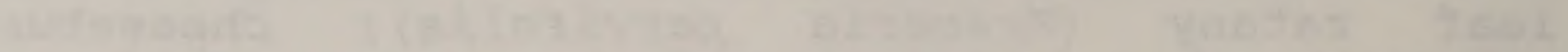

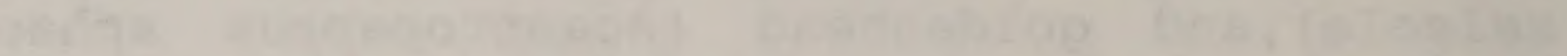

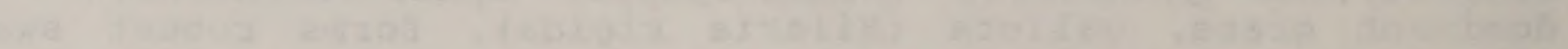

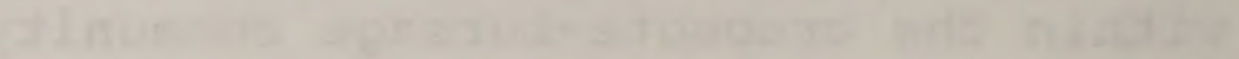


fasciculata). A partial list of these species occurring in Piute Valley is given in Table 1. The plant inventory concentrated efforts on sensitive plant species and perennial species for vegetation description, thus only a part of annuals and forbs which occur the Piute Valley is listed here. Plant synonymy follows Kartesz and Kartesz (1980) and Kartesz (1988).

\section{Endangered, Threatened, or Sensitive plants}

A rare and sensitive plant inventory was undertaken in the Piute Valley TMA between April and June, 1992 to determine the distribution and abundance of Federal candidate taxa identified for potential listing as threatened or endangered. Additionally, sensitive plant taxa of state or local interest were also inventoried. A list of the current federal (USFWS), State (Nevada Division of Forestry), and Northern Nevada Native Plant Society (NNNPS) status for each taxon that was inventoried is presented Table 2. Plant status was compiled from BLM, 1991. The distribution of sensitive plant taxa in Piute Valley from past and present surveys is presented in Figure 2 and illustrations of candidate taxa appear in Appendix I. Plant taxa were systematically searched for, starting at the locations they were last reported and then surveying similar habitat. All plant species were recorded at each site and the dominants noted. The total area surveyed was 180,000 acres (see Figure 1 for boundaries). Voucher specimens collected were deposited at UNLV. Precipitation for spring, 1992 was above normal, with 10 inches recorded at searchlight.

only one $T \& E$ candidate, rosy two-toned beardtongue (Penstemon bicolor ssp. roseus) was recorded within the Piute valley TMA during this inventory. Currently, it is listed as a Category 2 candidate. The other two taxa in Table 2 which were recorded from Piute Valley during this inventory are considered federal noncandidates at this time, but remain sensitive either because of their limited distribution (Cryptantha tumulosa) or because they are protected under Nevada Revised Statute 527.060 et seq., the Cactus and Yucca Law (Ferocactus acanthodes var. lecontei) (see Fig. 2).

Penstemon bicolor ssp. roseus occurs in shallow, coarse, gravelly washes in Clark and Nye Counties, Nevada. It occurs within the mixed Mojave shrubland community and the associated wash species include cat-claw, cheesebush, California buckwheat, and milkweed (Asclepias erosa). Subspecies roseus appears to be slightly more common than the other Candidate 2 subspecies, $P$. bicolor ssp. bicolor, which has light yellow flowers instead of pinkish. 

Table 1. Plant species observed in Piute valley. Relative abundance is as follows: $C=$ common, widespread throughout community type(s) or a community dominant; $0=$ occasional, scattered throughout community type(s) or locally abundant; $R=$ rare, few seen anywhere. Introduced taxa are shown by "*".

Species

Common Name

Relative Abundance

\section{SHRUBS}

Acacia greggii

Acamptopappus sphaerocephalus

Ambrosia dumosa

A. eriocentra

Amsonia tomentosa var. brevifolia

Androstephium brevifolium

Artemesia ludoviciana

Asclepias subulata

Aster sp.

Atriplex canescens

Bebbia juncea

Brickellia incana

B. californica

Ceratoides lanata

Chilopsis linearis

Chrysothamnus paniculatus

Coloeogyne ramosissima

Dyssodia cooperi

Encelia virginensis

E. frutescens

E. farinosa

Ephedra nevadensis

E. torreyana

E. viridis

Eriogonum fasciculatum

Fallugia paradoxa

Grayia spinosa

Gutierrezia microcephala

Hymenoclea salsola

Juniperus osteosperma

Krameria grayi

K. parvifolia

Larrea tridentata

Lepidium fremontii catclaw acacia C

rayless goldenhead C

white bursage $C$

wooly bursage C

wooly amsonia $\quad \mathrm{R}$

western mugwort 0

rush milkweed $\mathrm{R}$

four-wing saltbush 0

sweetbush 0

wooly brickell-bush 0

California

brickel1-bush 0

winterfat

desert willow 0

rabbitbrush C

blackbrush C

Cooper's dyssodia o

Virgin River encelia C

bush encelia 0

brittlebush R

Nevada ephedra C

Mormon tea 0

green ephedra 0

California buckwheat C

cliff rose 0

spiny hop-sage 0

snakeweed 0

cheesebush C

Utah juniper $O$

white ratany $\mathrm{C}$

little-leaf ratany C

creosotebush C

desert peppergrass 0 

Table 1. (cont.)

Lycium andersonii

L. cooper $i$

Menadora spinescens

Monardella linoides ssp. linoides

Nolina bigelovii

Petalonyx thurberi ssp. thurberi

Porophyllum gracile

Prunus fasciculata

Psilostrope cooperi

Quercus turbinella

Salazaria mexicana

Salvia dorrii

S. mohavensis

Sphaeralcea ambigua

stephanomeria pauciflora

Tetradymia canescens

T. spinosa var. longispina

Thamnosma montana

Vigueria deltoidea

Xylorhiza tortifolia

Yucca baccata

Y. brevifolia

Y. schidigera

\section{GRASSES}

Aristida purpurea

Bouteloua curtipendula

B. eriopoda

Bromus rubens*

Erioneuron pulchellum

Hilaria jamesii

H. rigida

Muhlenbergia porteri

oryzopsis hymenoides

Schismus barbata*

sitanion jubatum

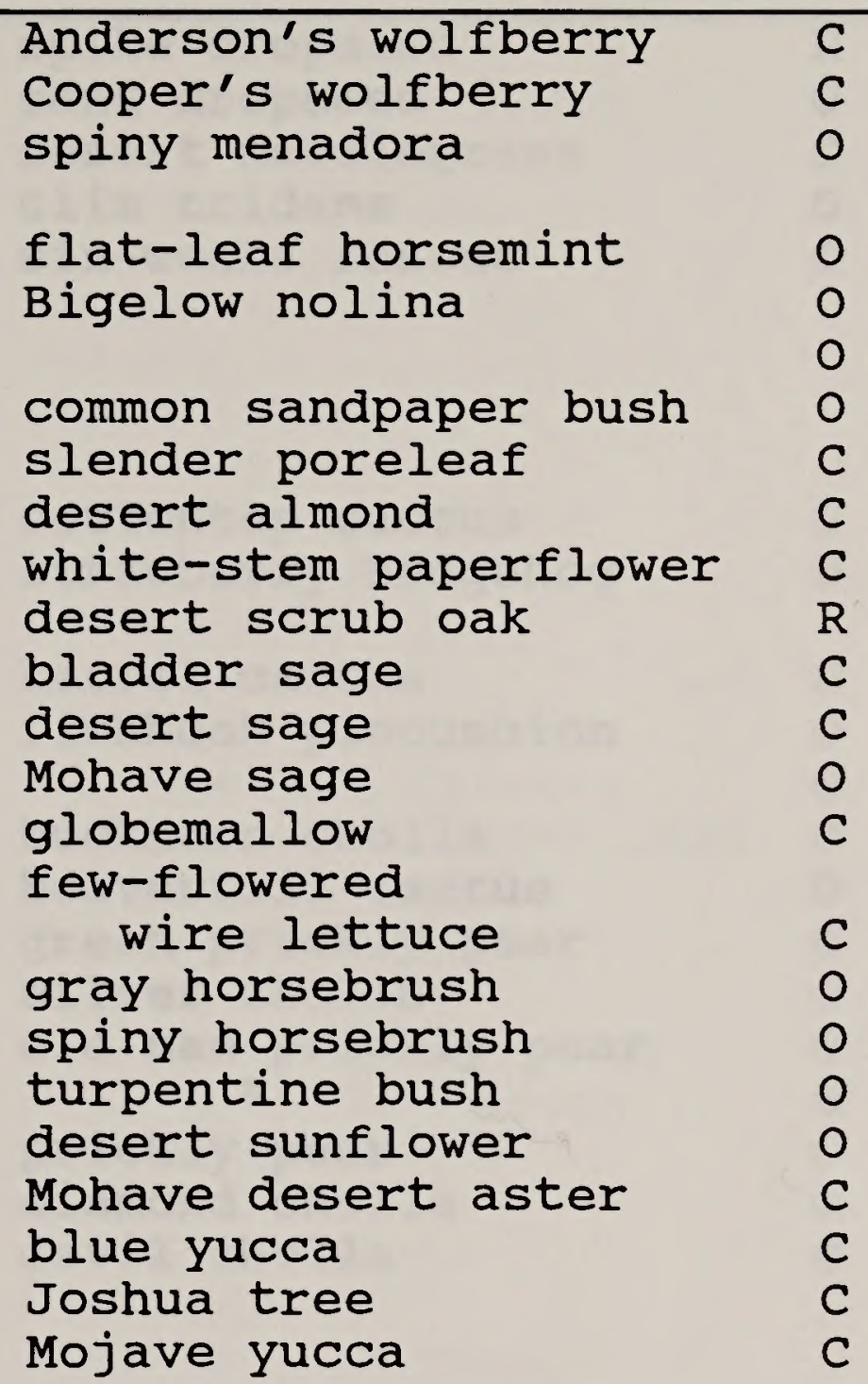

purple threeawn

side-oats grama

black grama

red brome

fluffgrass

curly galleta

big galleta

bush muhly

Indian ricegrass

Mediterranean grass

big squirreltail grass

0

O

C

C

C

O

C

C

O

C

O 

Table 1. (cont.)

species

Common Name

Relative

Abundance

Sporobolis contractus

spike dropseed

sand dropseed

$\mathrm{R}$

Sporobolis cryptandrus

Stipa speciosa

Tridens muticus

Vulpia octoflora*

desert needlegrass

slim tridens

six-weeks fescue

O

C

0

C

CACTI

Echinocactus polycephalus Echinocereus engelmannii

Ferocactus acanthodes var.lecontei

Mammillaria tetrancistra

Neolloydia johnsonii

opuntia acanthocarpa

0 . basilaris

o. chlorotica

o. echinocarpa

o. erinacea var. ursina

o. erinacea var. erinacea

o. phaeacantha var. major

o. ramosissima

o. stanleyi var. parishii

cottontop cactus

strawberry hedgehog

0

0

barrel cactus

0

fishhook pincushion 0

buckhorn cholla

0

beavertail cactus

C

green prickly pear

silver cholla

old man prickly pear

0

0

0

0

prickly pear

diamond cholla

0

0

devil cholla

0

0

\section{HERBACEOUS FORBS}

Allium sp.

Amaranthus fimbriatus

Antheropeas wallacei

Argythamnia neomexicana

Amsinkia tessellata

Arabis spp.

Arenaria fendleri

Asclepias nyctaginifolia

A. erosa

Astragalus layneae

A. lentiginosus

var. variabilis

Astragalus lentiginosus

var. fremontii

A. purshii var. lectulus desert onion

fringed amaranth

wooly daisy

common silverbush

fiddleneck

rockcress

Fendler sandwort

Mojave milkweed

desert milkweed

Layne's milkvetch

freckled milkvetch

Pursh's milkvetch
R

O

C

0

C

0

O

$\mathrm{R}$

$\mathrm{O}$

0

o

$\mathrm{O}$

R 


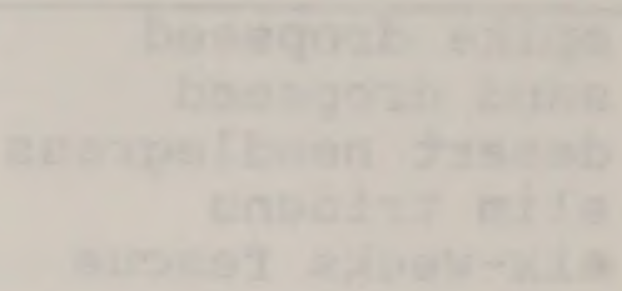

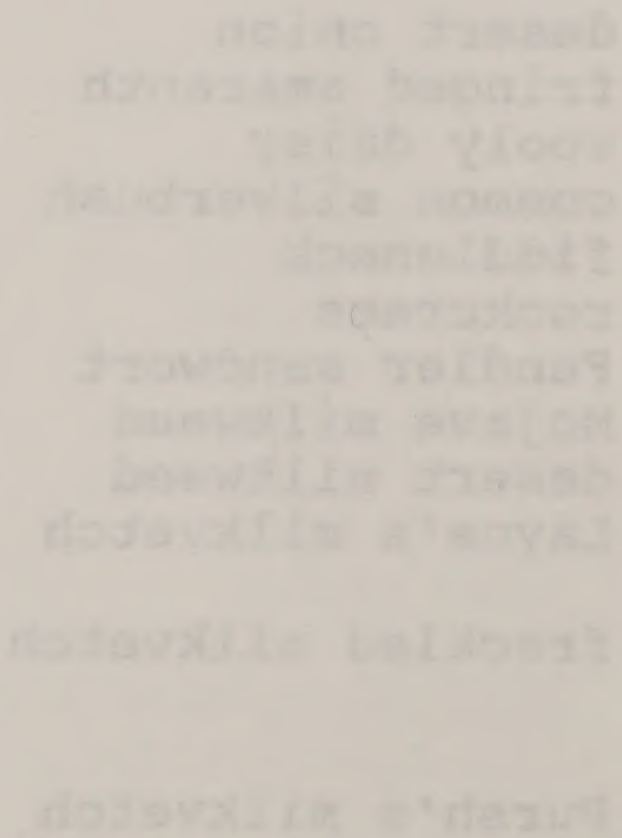

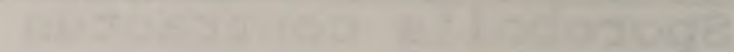

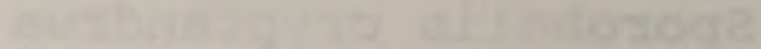

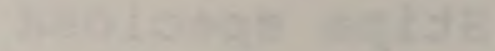

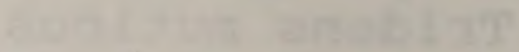

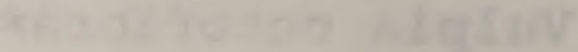

7. $3 \ln ^{2}$

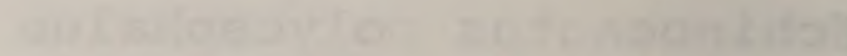

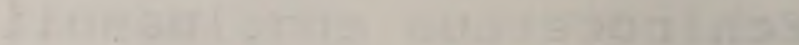

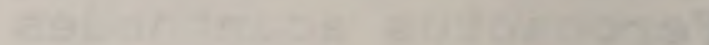

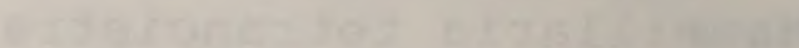

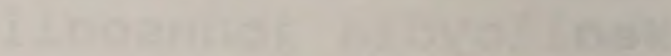

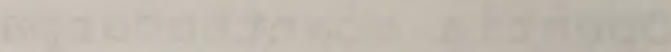

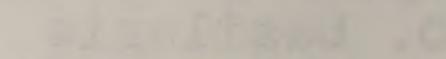

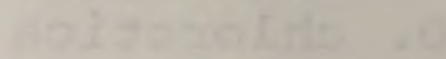

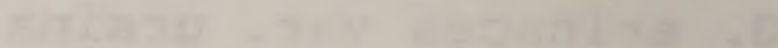

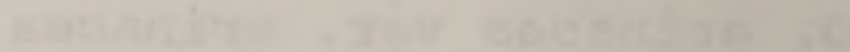

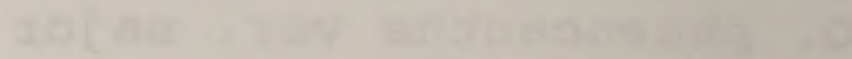
(15.

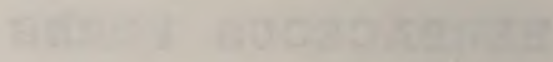

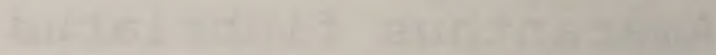

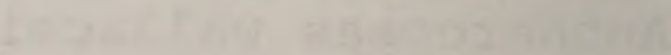

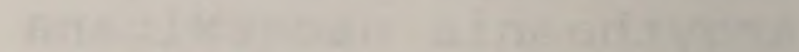

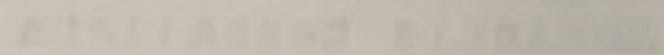

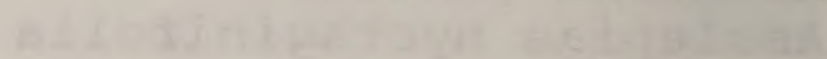

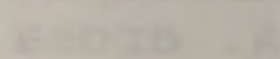

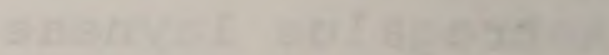

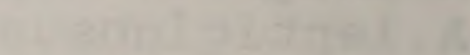

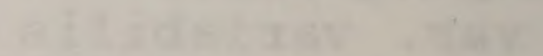

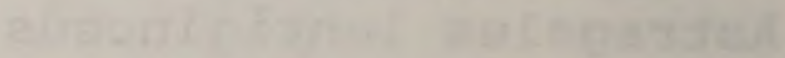

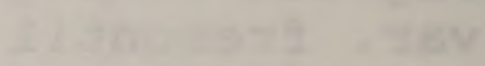

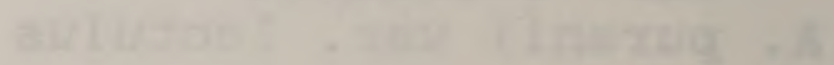


Baileya multiradiata Calochortus flexuosus C. kennedyi

Calycoseris parryi Camissonia brevipes Castilleja chromosa Chaenactis fremontii C. macrantha

Chaenactis stevioides

Chamaesyce albomarginata Chorizanthe rigida

C. brevicornu

Cirsium neomexicanum Cryptantha angustifolia

C micrantha

c. tumulosa

Cucurbita palmata

Cuscuta denticulata

Dalea mollissima

Datura metalloides

Delphinium parishii

Descurania pinnata

Dichlostemma pulchella

Dyssodia pentaschaeta

Eriastrum diffusum

Erigeron concinnus

Eriogonum deflexum

E. inflatum

E. maculatum

E. nidularium

E. plumatella

E. thomasii

E. trichopes

Erodium cicutarium*

Eschscholzia glyptosperma

E. minutiflora

Gaura coccinea

Gilia spp.

Langloisia setosissima

L. schottii

Lepidium fremontii

Linanthus aureus

Lotus tomentellus desert marigold

strangling mariposa

desert mariposa

yellow tackstem

yellow cups

desert paintbrush

Fremont's pincushion

large-flowered

pincushion

broad-flowered

pincushion

rattlesnake weed

spiny herb

brittle chorizanthe

New Mexican thistle

creosotebush cryptantha

redfoot cryptantha

Mojave cryptantha

coyote melon

dodder

silk prairie clover 0

sacred datura 0

larkspur 0

tansy mustard C

bluedick 0

fire-hair dogweed C

spreading eriastrum C

hairy fleabane $R$

skeleton weed C

desert trumpet $C$

birdnest buckwheat $\quad C$

flat-top buckwheat 0

Thomas's buckwheat 0

little trumpet buckwheat $c$

filaree

desert poppy

little desert poppy C

scarlet gaura 0

bristly langloisia C

Schott's langloisia o

desert peppergrass 0

desert gold

desert birdfoot deervetch

0

C

C

C

0

C
C

deervetch C

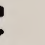

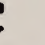

.

.

,

.

.

.

.

0
0
0
$C$
$C$
0

0
0
$C$




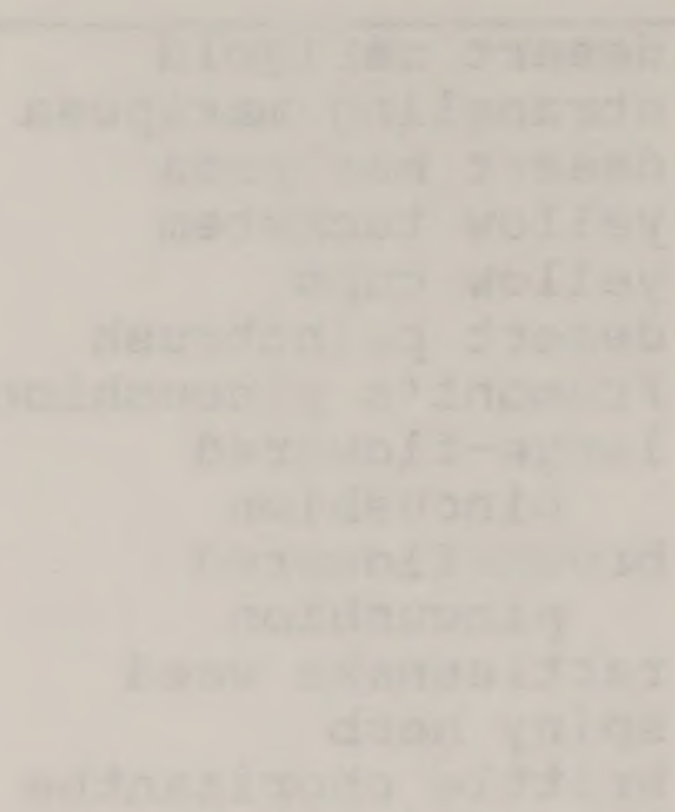

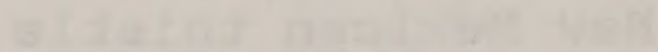

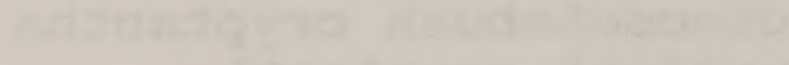

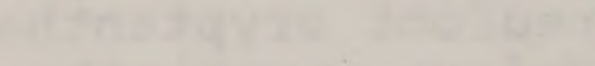

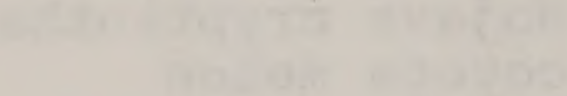
(1) (1) 4

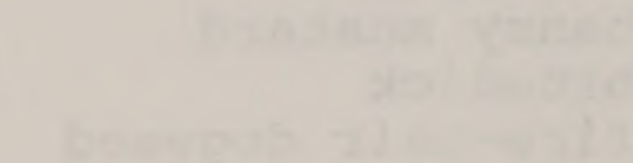

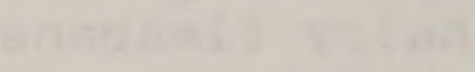

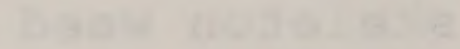

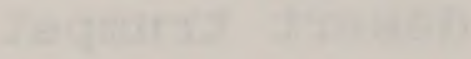

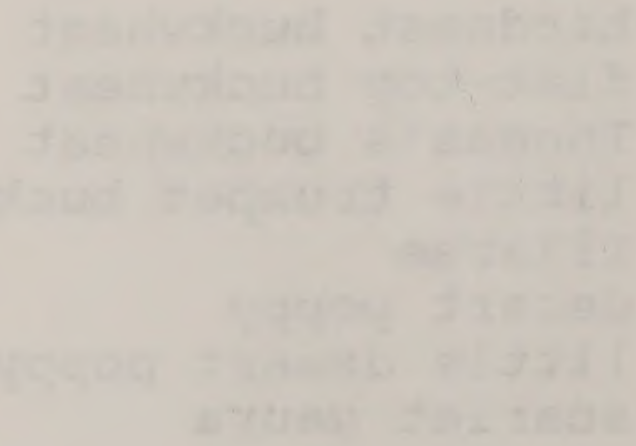

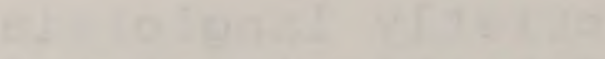

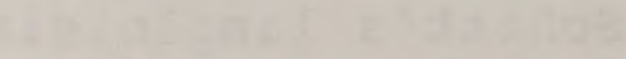

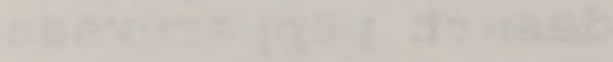

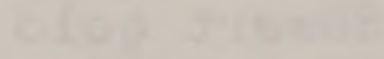

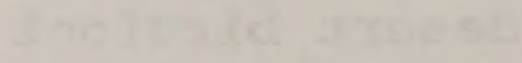

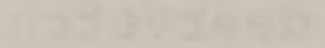

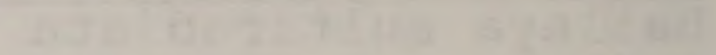

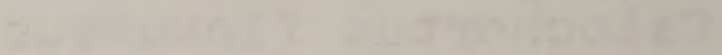

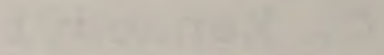

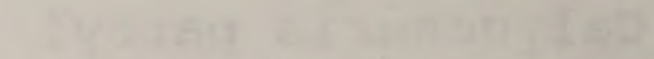

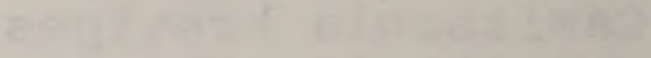
C.

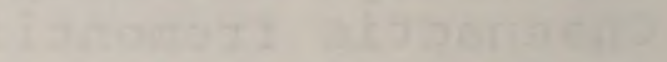

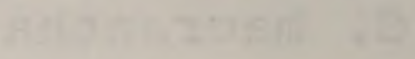

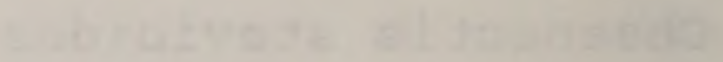

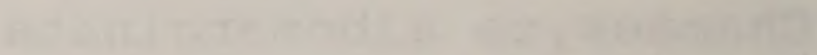

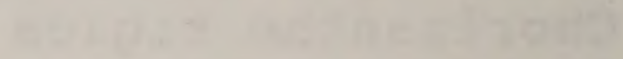

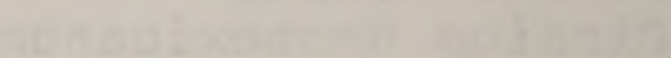

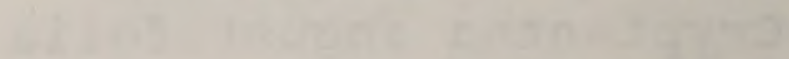

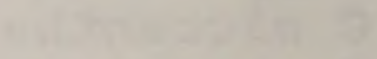

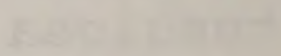

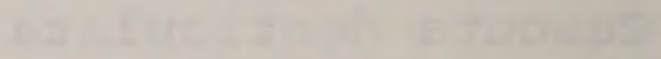

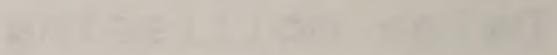

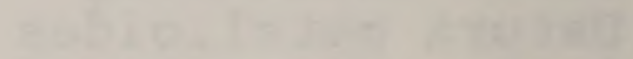

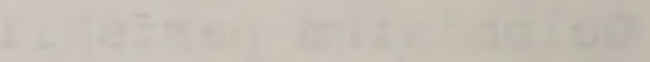

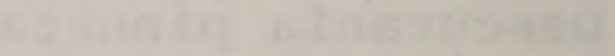

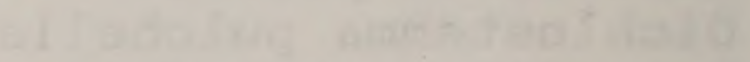

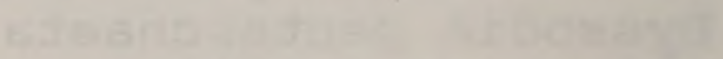

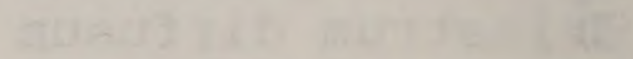

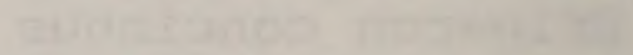

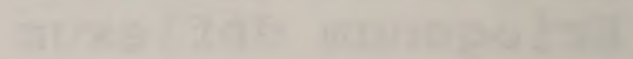

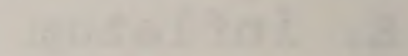

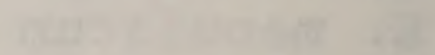

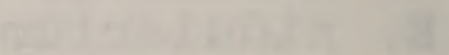

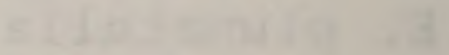
(3)

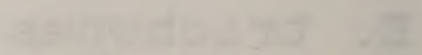

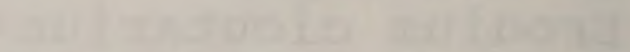

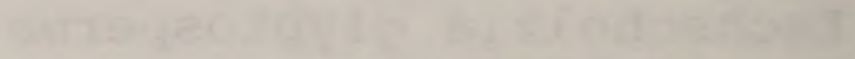

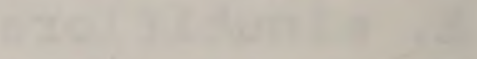

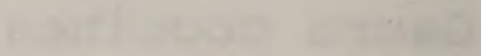

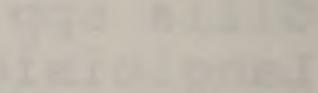

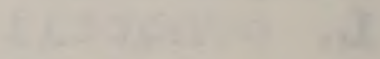

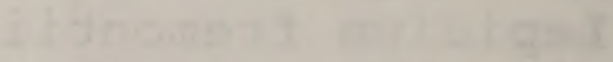

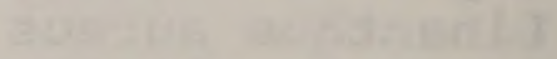

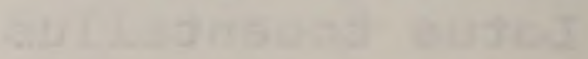


Table 1. (cont.)

\begin{tabular}{|c|c|c|}
\hline Lotus rigidus & desert deervetch & 0 \\
\hline Lupinus sp. & & 0 \\
\hline L. arizonicus & Arizona lupine & C \\
\hline $\begin{array}{l}\text { L. concinnus ssp. orcuttii } \\
\text { Malacothrix californica }\end{array}$ & elegant lupine & C \\
\hline \multicolumn{2}{|l|}{ Malacothrix californica } & \\
\hline M. coulteri & snakehead & 0 \\
\hline Marrubium vulgare* & horehound & $\mathrm{R}$ \\
\hline Menadora scabra & rough menadora & 0 \\
\hline Mentzelia tricuspis & desert stickleaf & $\mathrm{R}$ \\
\hline Mirabilis incarnata & windmills & C \\
\hline \multicolumn{3}{|l|}{ M. multiflora } \\
\hline M. coccineus & $\begin{array}{l}\text { many-rlower Iour-o'clock } \\
\text { scarlet four-o'clock }\end{array}$ & $\mathrm{R}$ \\
\hline M. bigelovii & wishbone bush & C \\
\hline Orobanche cooperi & Cooper's broomrape & $\mathrm{R}$ \\
\hline Pectocarya recurva & recurve pectocarya & c \\
\hline Penstemon bicolor ssp. roseus & rosy two-tone penstemon & 0 \\
\hline Phacelia distans & common phacelia & 0 \\
\hline P. crenulata & notch-leave phacelia & C \\
\hline P. distans & & 0 \\
\hline P. fremontii & Fremont phacelia & \\
\hline \multicolumn{3}{|l|}{$\begin{array}{l}\text { P. parishii } \\
\text { P. rotundifolia }\end{array}$} \\
\hline \multicolumn{3}{|l|}{ Physalis crassifolia } \\
\hline var. crassifolia & thick-leaf groundcherry & 0 \\
\hline Plantago insularis & wooly plantain & c \\
\hline P. purshii & Pursh plantain & C \\
\hline Quincula lobata & purple groundcherry & $\mathrm{R}$ \\
\hline Raphinesquia neomexicana & desert chicory & C \\
\hline Salsola iberica* & Russian thistle & C \\
\hline Salvia columbariae & chia & C \\
\hline stanleya pinnata ssp. pinnata & prince's plume & 0 \\
\hline Stephanomeria exigua & small wire lettuce & C \\
\hline Streptanthella longirostrus & long-beak twistflower & c \\
\hline Sysimbrium altissimum* & tumble mustard & C \\
\hline Tiquilia canescens & shrubby tiquilia & 0 \\
\hline Tragia ramosa & & $\mathrm{R}$ \\
\hline Verbena gooddingii & desert verbena & 0 \\
\hline
\end{tabular}


Table 2. Endangered, Threatened, or sensitive Plants reported from piute valley TMA.

\begin{tabular}{|c|c|c|c|c|}
\hline Species & $\begin{array}{l}\text { Federal }{ }^{1} \\
\text { status }\end{array}$ & $\begin{array}{l}\text { Nevada }^{2} \\
\text { status }\end{array}$ & $\begin{array}{l}\text { NNNPS }^{3} \\
\text { status }\end{array}$ & $\begin{array}{r}\text { Last } \\
\text { reported } \\
\text { occurrence }\end{array}$ \\
\hline $\begin{array}{l}\text { bear claw poppy } \\
\text { (Arctomecon } \\
\text { californicum) }\end{array}$ & $\mathrm{C} 2$ & $\bar{E}$ & $\mathrm{~T}$ & 1906 \\
\hline $\begin{array}{l}\text { chalk live-forever } \\
\text { (Dudleya pulverulenta } \\
\text { ssp. arizonica) }\end{array}$ & - & - & OR & 1966 \\
\hline $\begin{array}{l}\text { pink two-tone penstemon } \\
\text { (Penstemon bicolor } \\
\text { var. roseus) }\end{array}$ & C2 & - & W & 1992 \\
\hline $\begin{array}{l}\text { barrel cactus } \\
\text { (Ferocactus } \\
\text { acanthodes } \\
\text { var. lecontei) }\end{array}$ & $3 C$ & E & W & 1992 \\
\hline $\begin{array}{l}\text { Mojave cryptantha } \\
\text { (Cryptantha tumulosa) }\end{array}$ & $3 C$ & - & W & 1992 \\
\hline $\begin{array}{l}\text { Clokey pincushion } \\
\text { (Coryphantha vivipara } \\
\text { ssp. rosea) }\end{array}$ & $3 c$ & $\mathrm{E}$ & D & 1973 \\
\hline
\end{tabular}

1Federal Status codes (FR, 1985, Vol.50, No. 188)

C2 Candidate 2 - USFWS needs more information before listing is possible.

3C Non-candidate - taxa that have proven to be more widespread than previously believed and/or have no identifiable threat, but which may be re-evaluated.

${ }^{2}$ Nevada status codes:

E Protected by Nevada Revised Statute $527 \mathrm{j} .270$ as a critically endangered plant, or by NRS 527.060 under the Cacti and Yucca Law. - No status has been given.

${ }^{3}$ NNNPS (Northern Nevada Native Plant Society)

w Watch - taxa of uncertain abundance and distribution, and/or those whose threats cannot be defined.

OR Other Rare - taxa of limited distribution.

D Deletion - indicates taxa to be more widespread than originally thought, and/or no identifiable threat.

+Dates for reported occurrence before this 1992 inventory are from Nevada Natural Heritage Program (1991). 


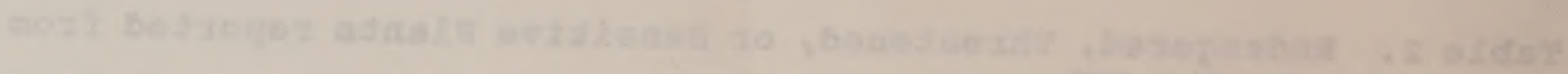
- wathes

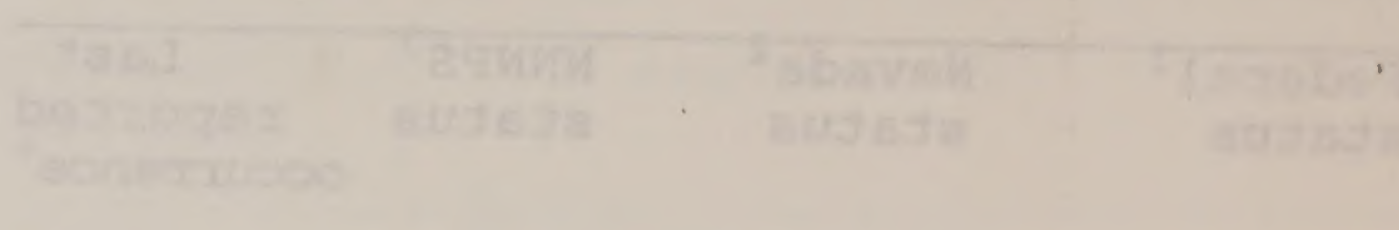
7T

201907.

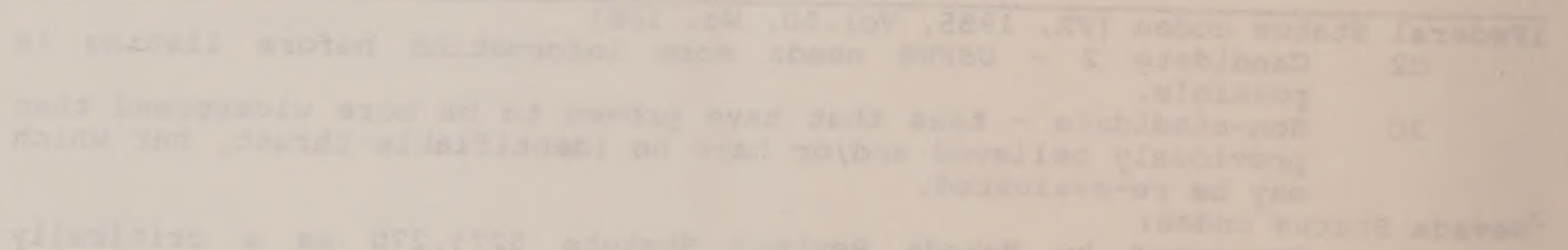

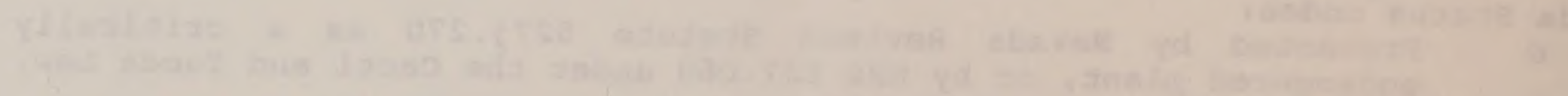

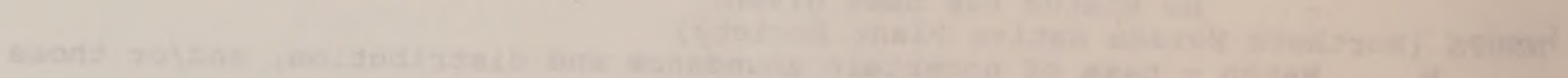

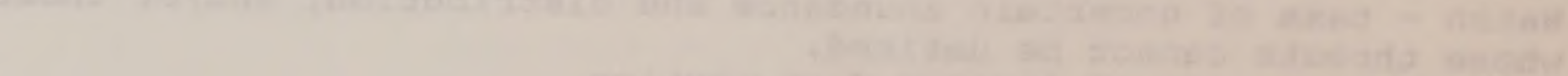

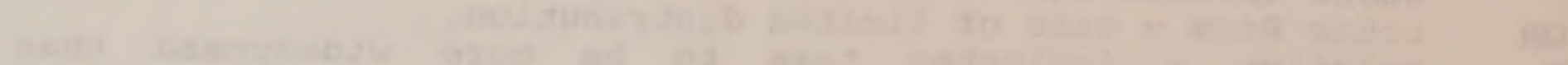

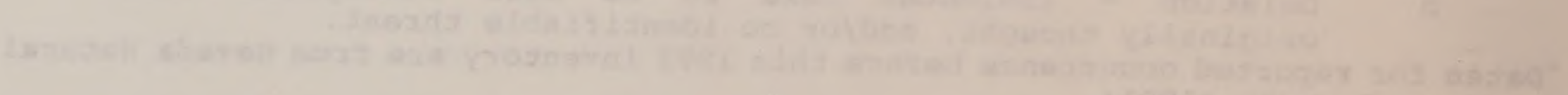

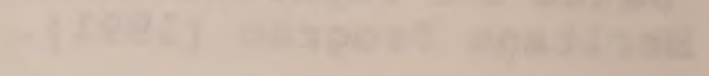




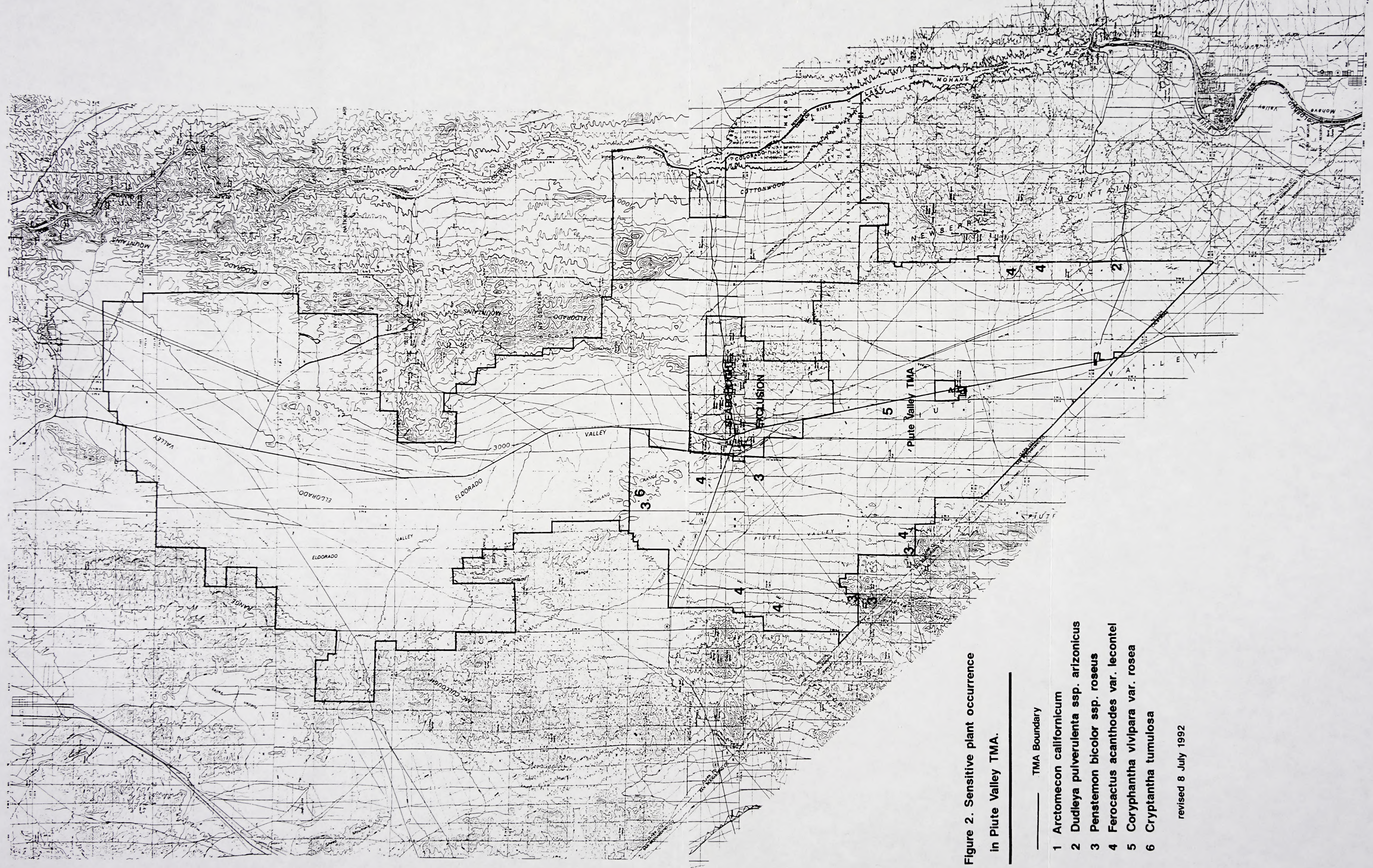



Known locations in the Piute Valley TMA include:

1 T28S, R63E, Secs. 1, 7, $1160 \mathrm{~m}, 41$ individuals;

2 T29S, R63E, Secs. 4, 32, 1120 m, 15 individuals;

3 T30S, R62E, Sec. 15, $1120 \mathrm{~m}, 1$ individual.

On the west side of Piute Valley but outside the boundary of the TMA, scattered populations of bicolored penstemon were reported at the following locations:

T30S, R62E, Secs. 9, 4, 1100-1200 m, scattered individuals along roadside (approximately 70 individuals).

The distribution of Penstemon bicolor ssp. roseus in Piute valley is presented in Figure 3. Also included are sites which were surveyed for this species.

The Mojave cryptantha (Cryptantha tumulosa) occurs in rocky outcrops of Clark and Nye counties, usually in the Pinyon-Juniper community. It is currently a category $3 \mathrm{C}$ taxa, but is on the NNNPS watch list. It was located on an outcrop of decomposed granite in the southern foothills of the Highland Range within the boundaries of the TMA. The associated vegetation was scrub oak (Quercus turbinella), milk-vetch (Astragalus purshii var. lectulus), fishhook cactus (Mammillaria tetrancistra) and the cactus, Neolloydia johnsonii.

Barrel cactus (Ferocactus acanthodes var. lecontei) is common on hillsides throughout Piute Valley, where the soils are rocky, welldrained, and coarse. This cactus is also a category 3C. The dominant taxa associated with this cactus are Joshua tree, Mohave yucca, creosotebush, and often other cacti (e.g. Opuntia spp.).

One plant species that is noteworthy despite a lack of listing is the cactus, Neolloydia johnsonii. The Searchlight population is apparently the only one that displays a yellow flowered form; all other populations have pink to purple flowers. Searchlight is the type local for Neolloydia johnsonii.

Three taxa, bear claw poppy (Arctomecon californicum), chalk liveforever (Dudleya pulverulenta spp. arizonica), and Clokey pincushion (Coryphantha vivipara var. rosea) were looked for, but not found during the inventory. They have in the past been reported from Piute Valley (see Table 2 and Fig. 2). Of these, only the bear claw poppy is a federal $\mathrm{C} 2$ candidate species. It typically thrives in gypsum soils within the creosote-bursage community, but has not been seen in Piute Valley since the early 1900's.Further study of the flora of Piute Valley should be 


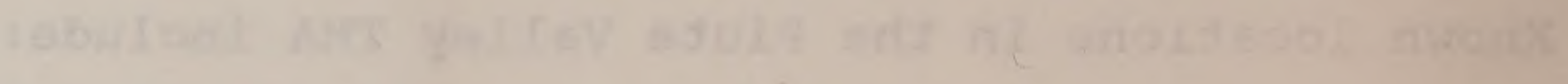

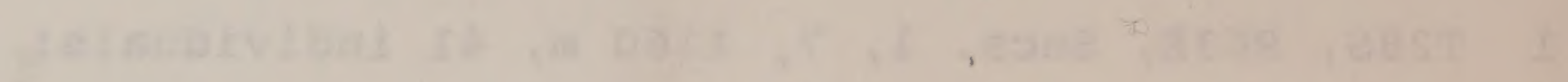

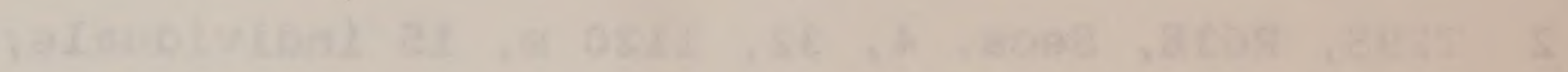

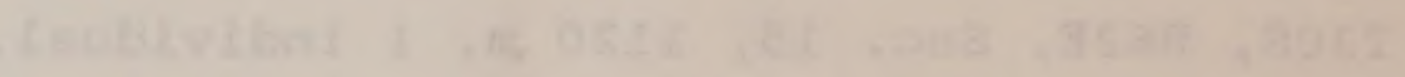

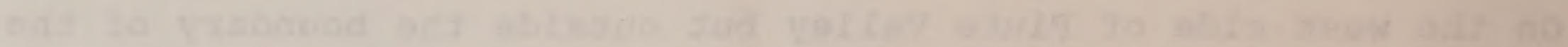

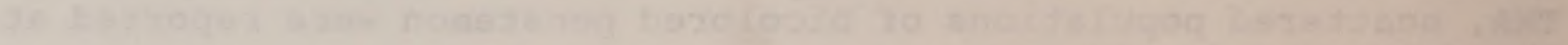

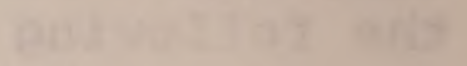

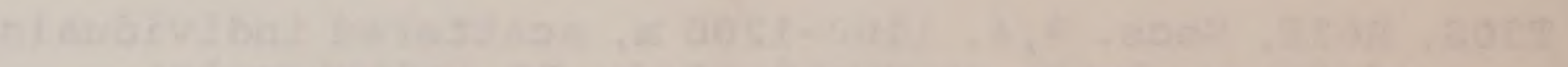

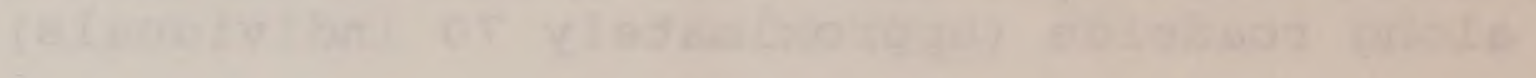

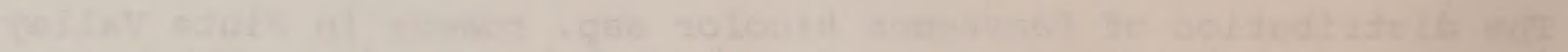

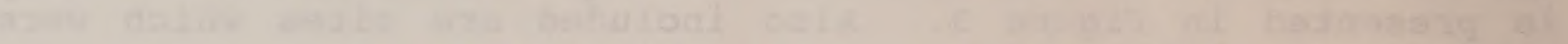
-

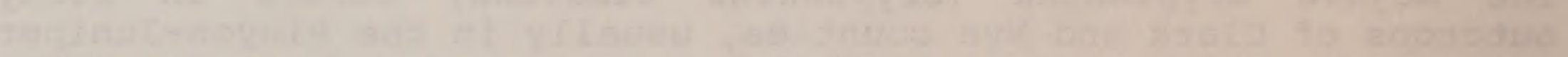

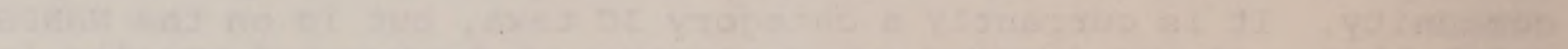

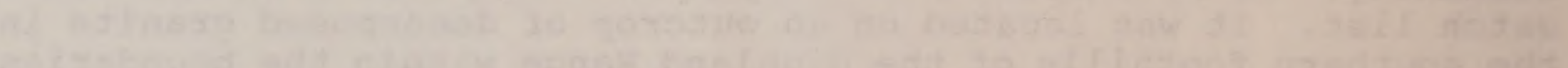

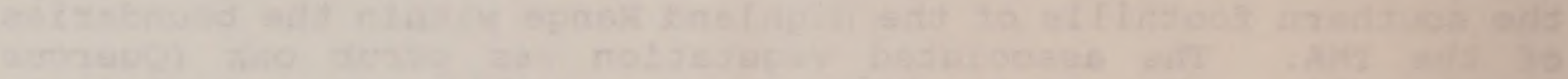

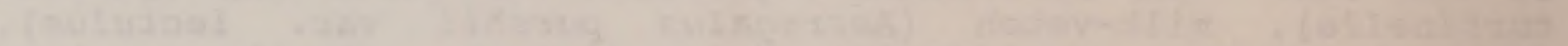

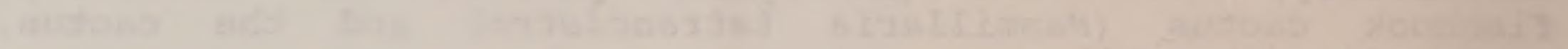

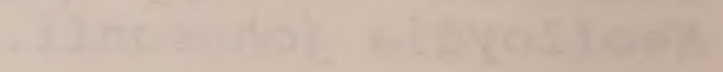

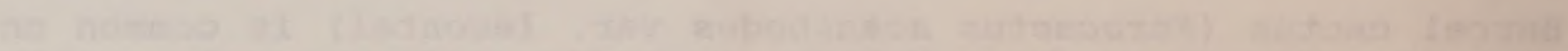

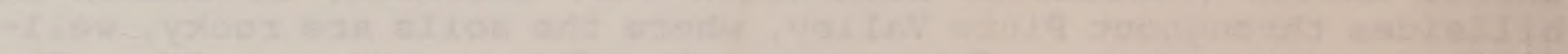

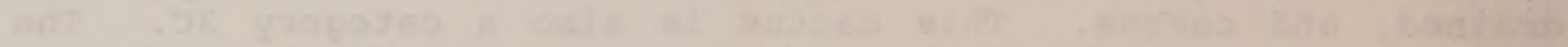

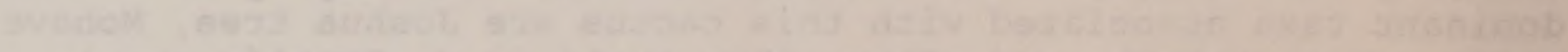

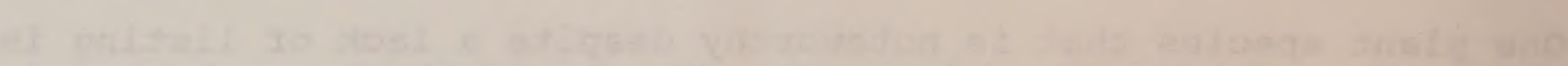

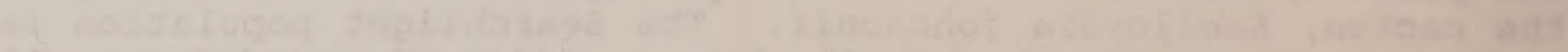

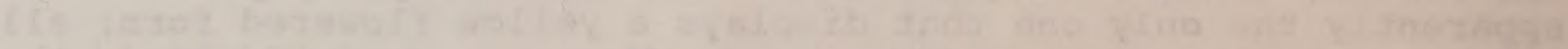

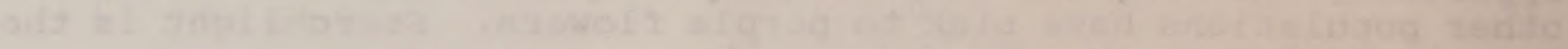

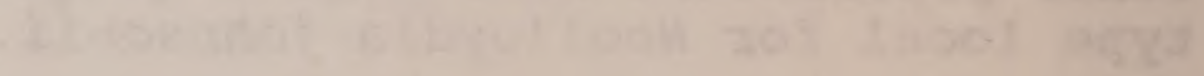

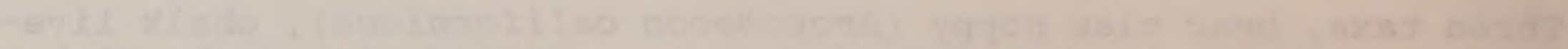

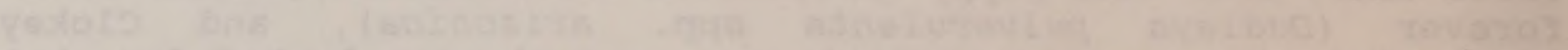

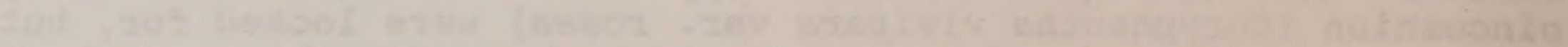

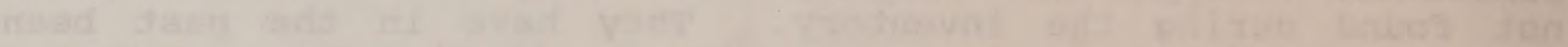

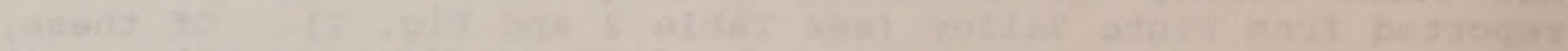
41.

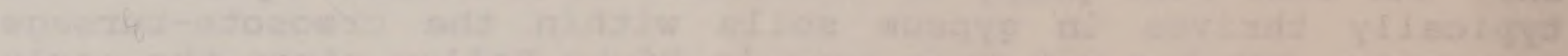

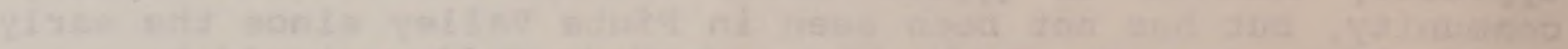

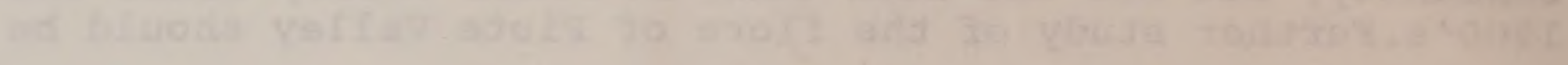




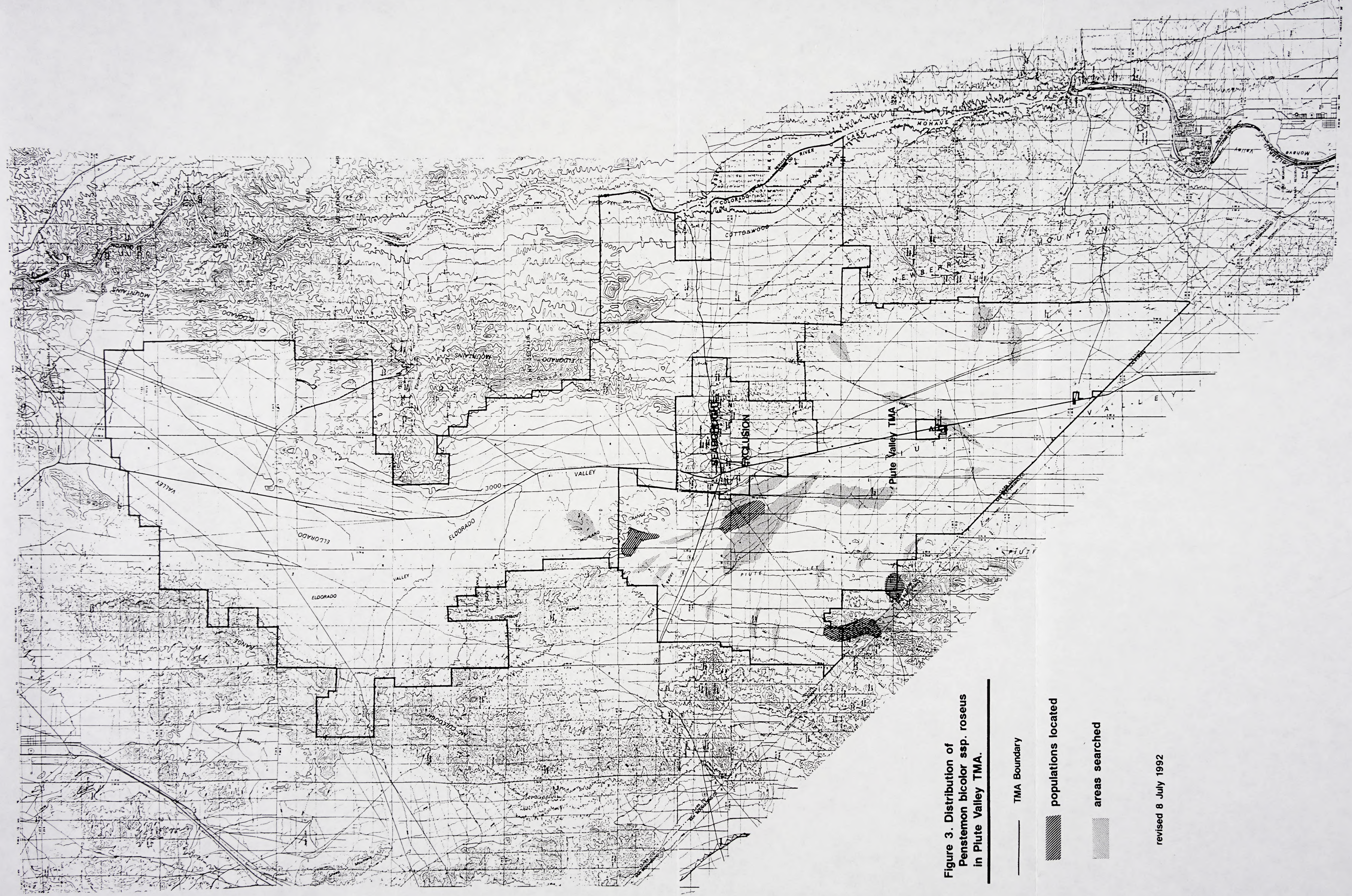



continued. Since it is the prototype of desert tortoise conservation areas, a full floristic and vegetation study should be performed to document all vascular plants and communities occurring in the valley.

\section{References}

BLM. 1991. Endangered, threatened, and sensitive vascular plants of Nevada. Nevada State Office, BLM, Reno.

Cronquist A, Holmgren AH, Holmgren NH, and Reveal JL. 1972. Intermountain flora, Vol. 1. Hafner, NY.

Kartesz J. and R. Kartesz. 1980. A synonymized checklist of the vascular flora of the United States, Canada, and Greenland. Vol. II. Univ. North Carolina Press. Chapel $\mathrm{Hill}$, NC.

Kartesz J. 1986. Flora of Nevada. Unpublished PhD dissertation. Reno, Nevada.

Mozingo, HN, and M Williams. 1980. Threatened and endangered plants of Nevada. An illustrated manual. BLM State Office, Reno, NV.

Nevada Natural Heritage Program. 1991. Sensitive species of Nevada, 1991 report. Nevada Natural Heritage Program, Reno, NV. 


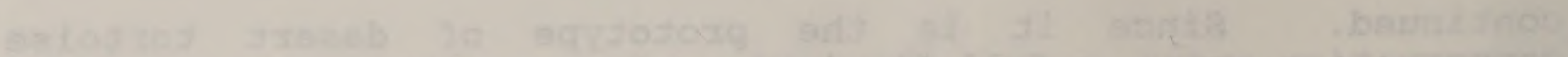

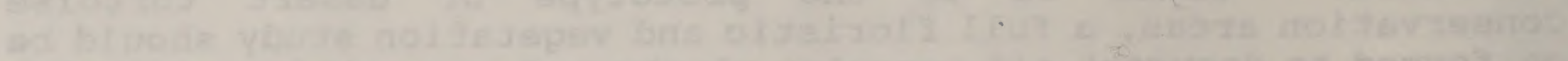

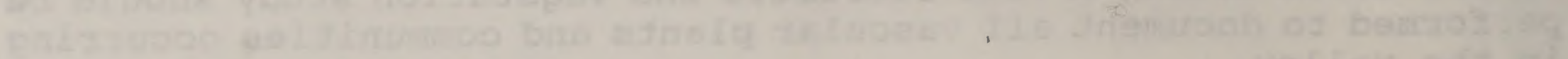

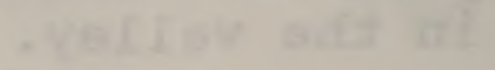

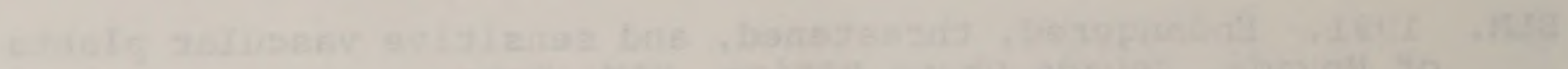

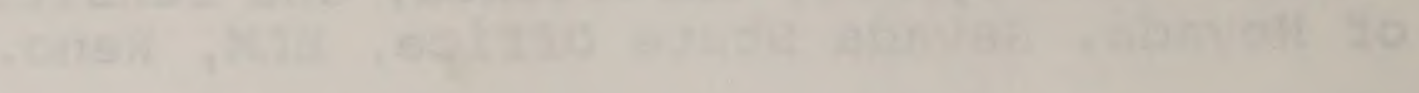

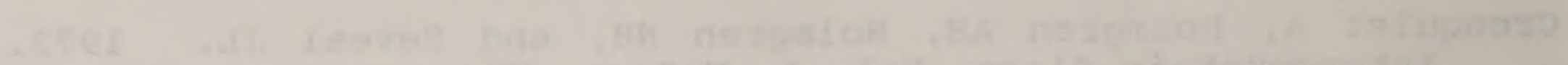

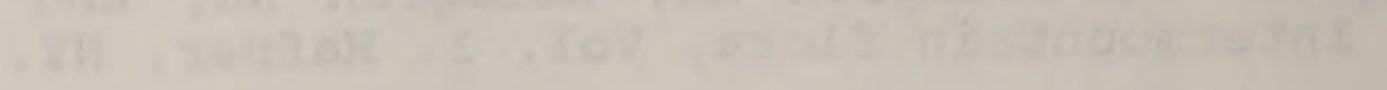

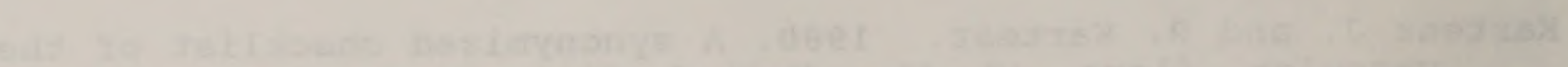

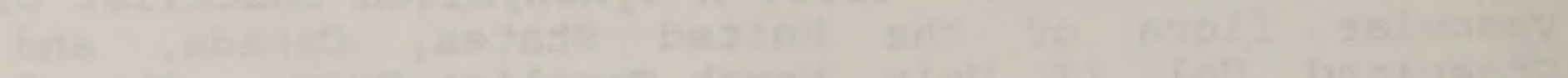
Catar.

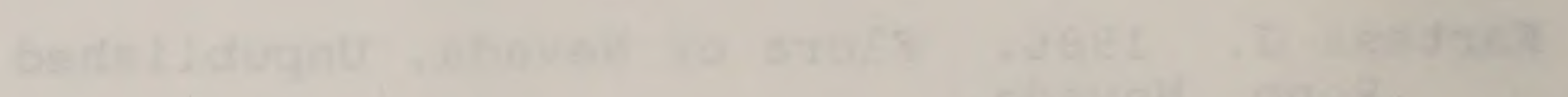

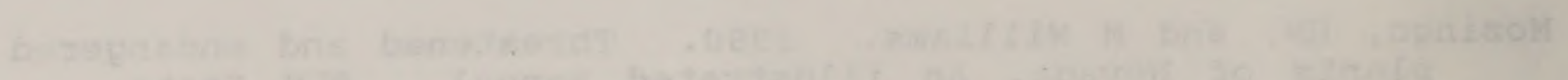

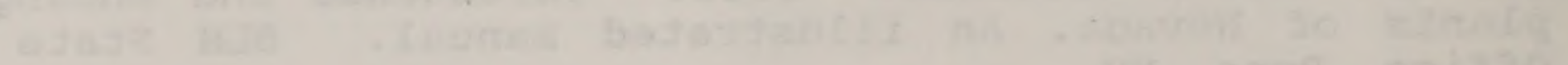

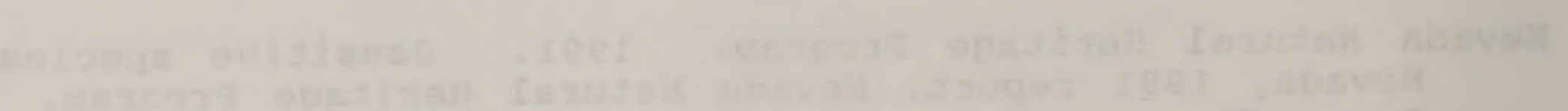

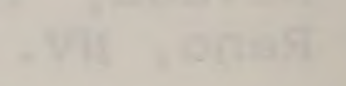




\section{Appendix 1. Candidate plant taxa (from Mozingo and Williams, 1980).}

ARCTOMECON - CALIFORNICA
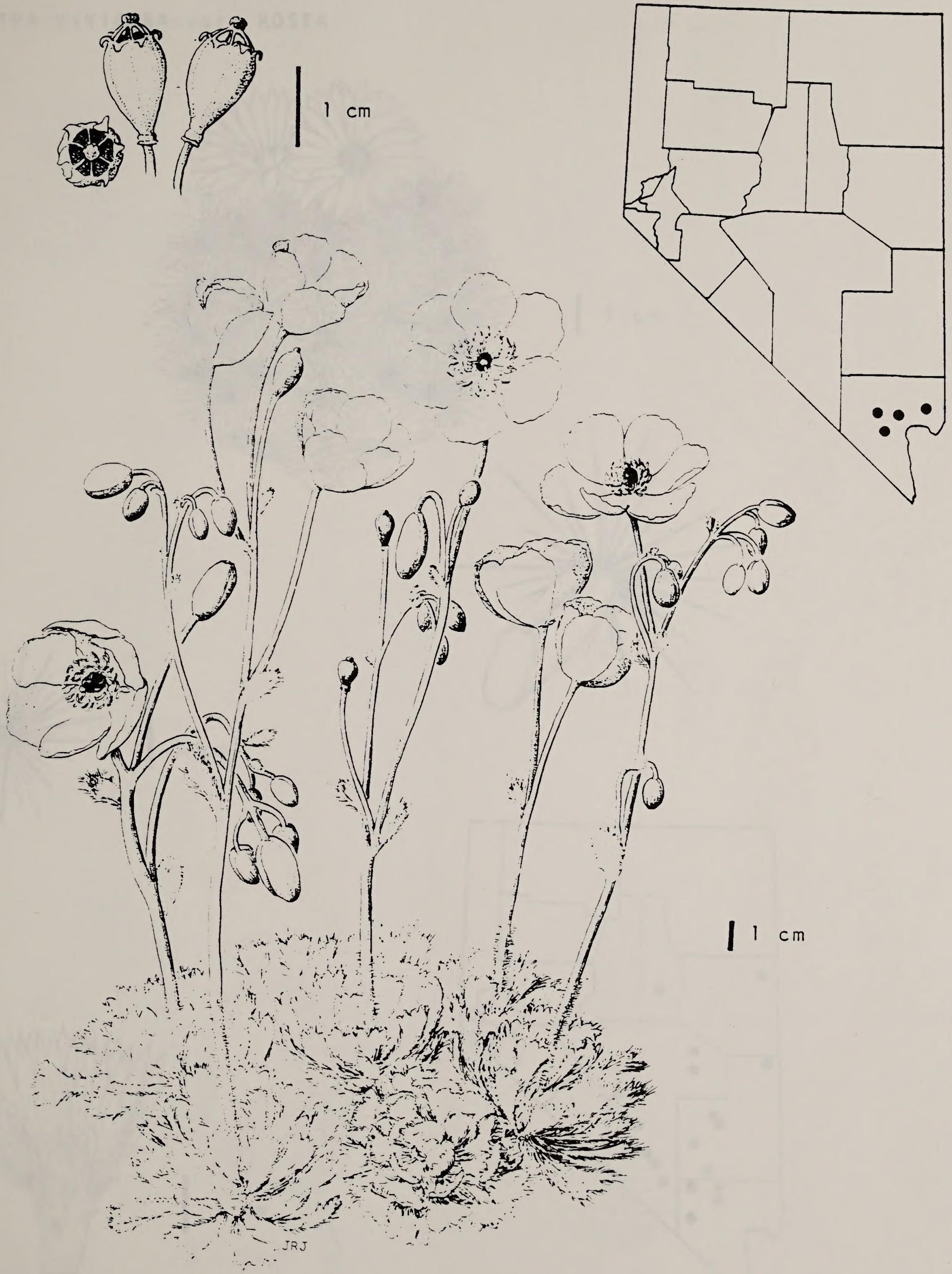


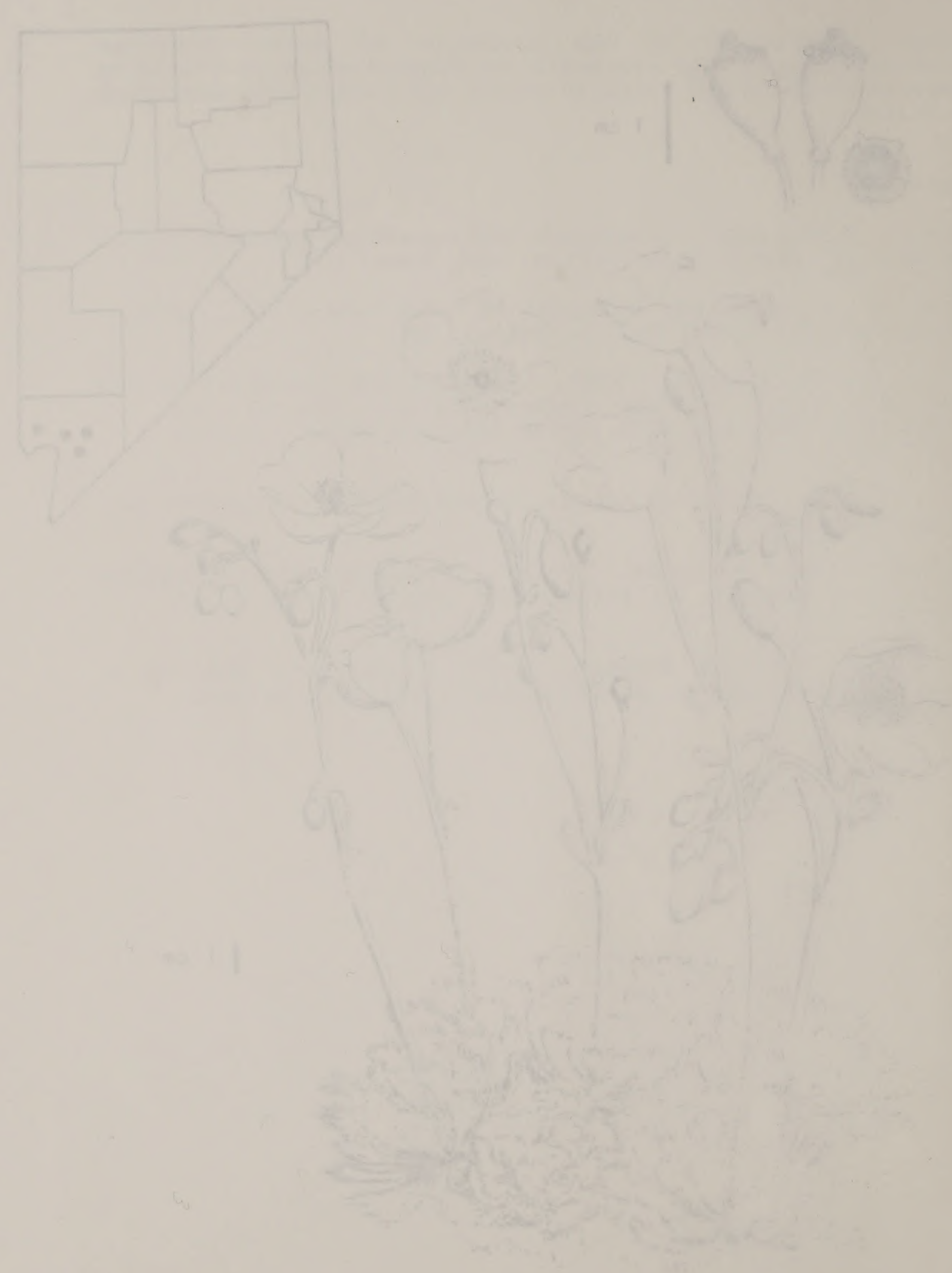


CORYPHANTHA VIVIPARA VAT. ROSEA
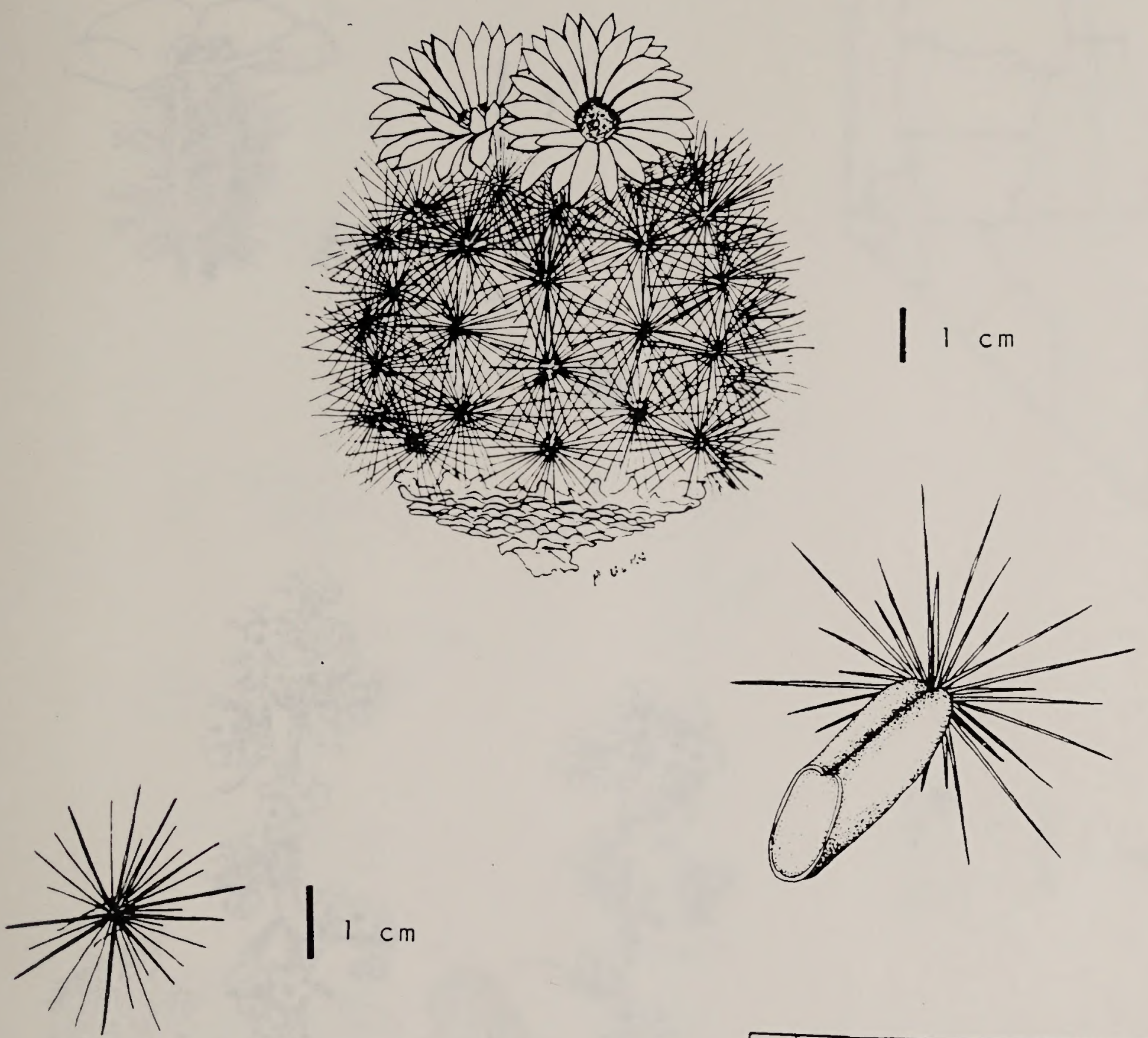

$1 \mathrm{~cm}$
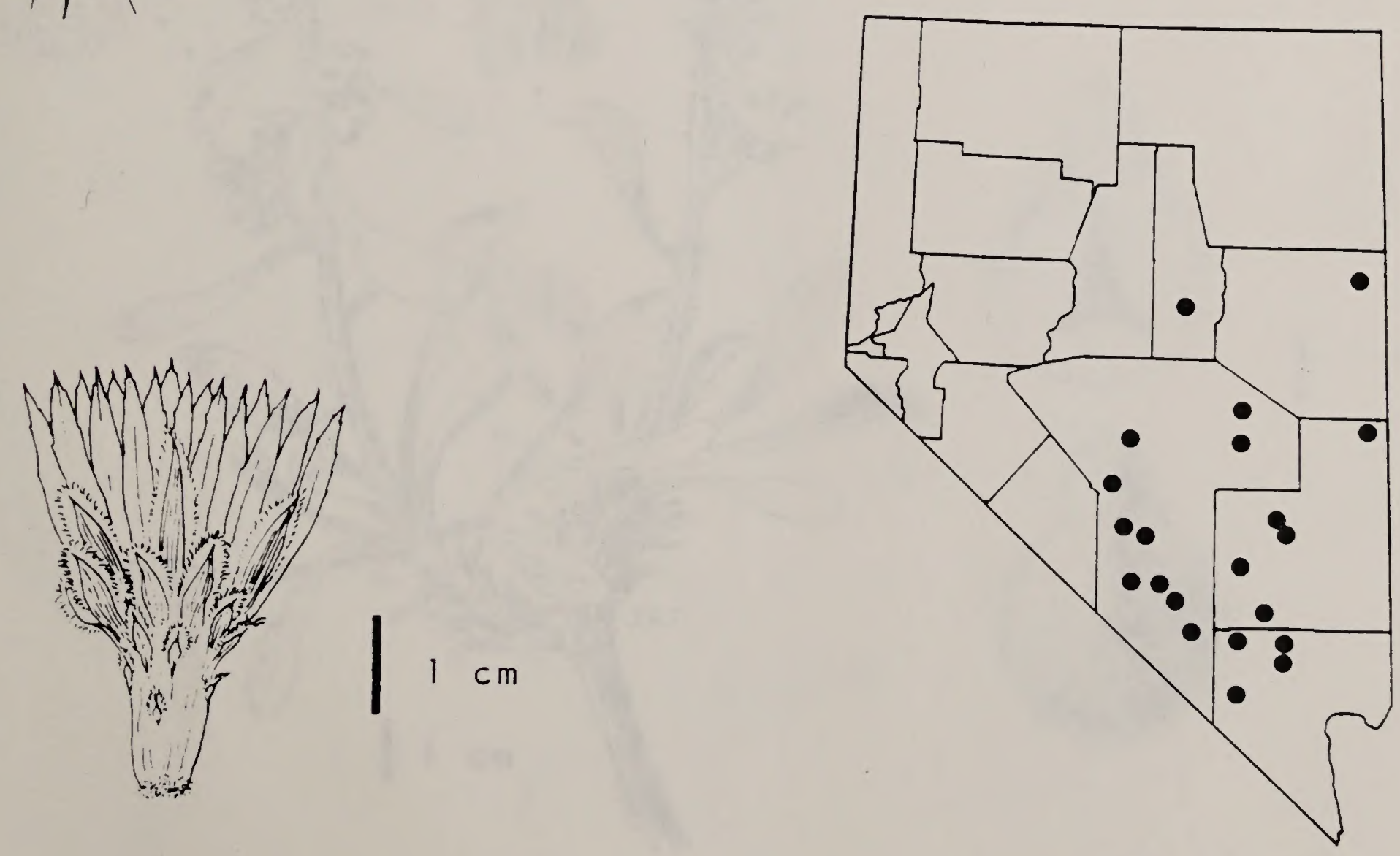

CRYPTANTHA TUMULOSA
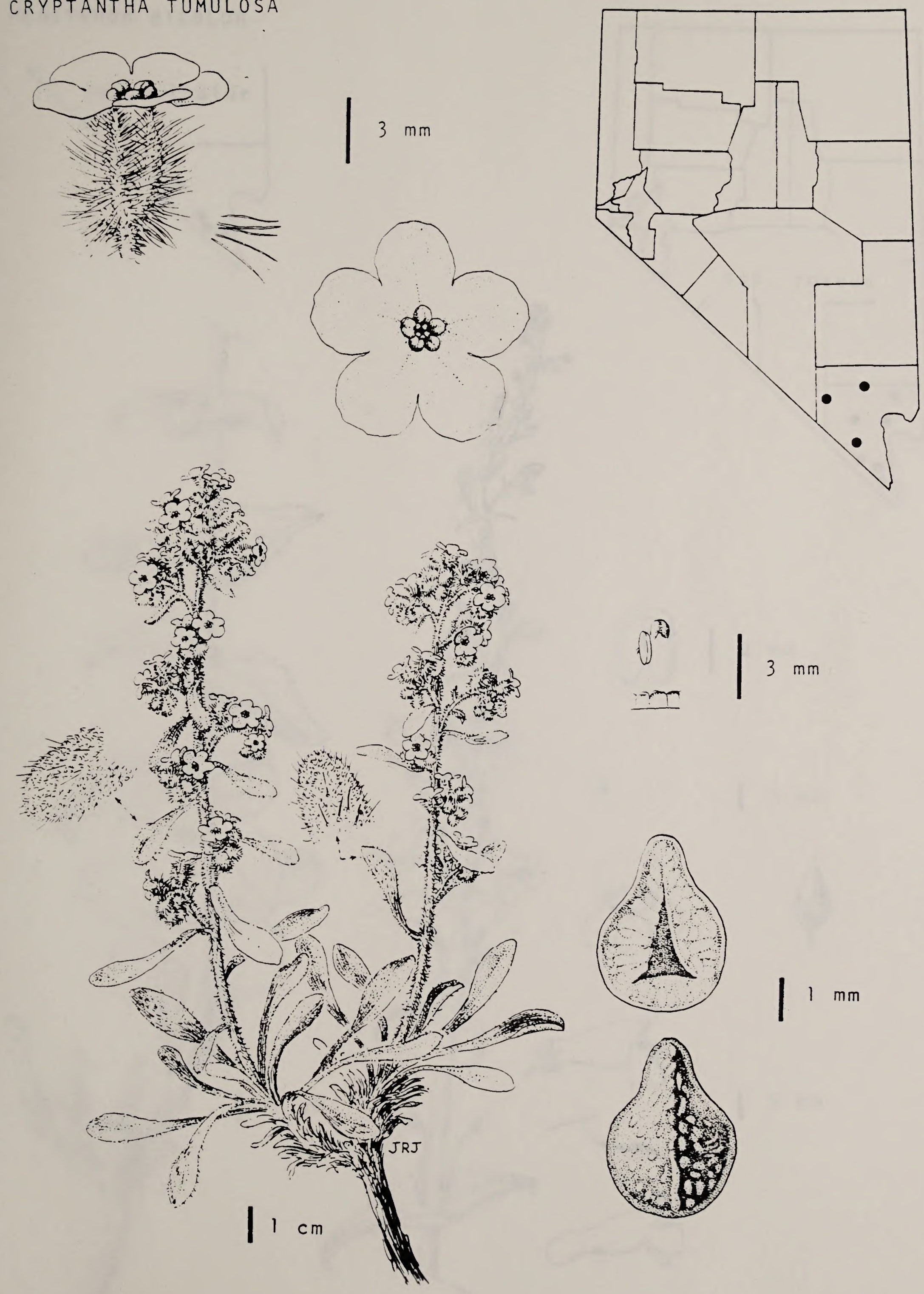


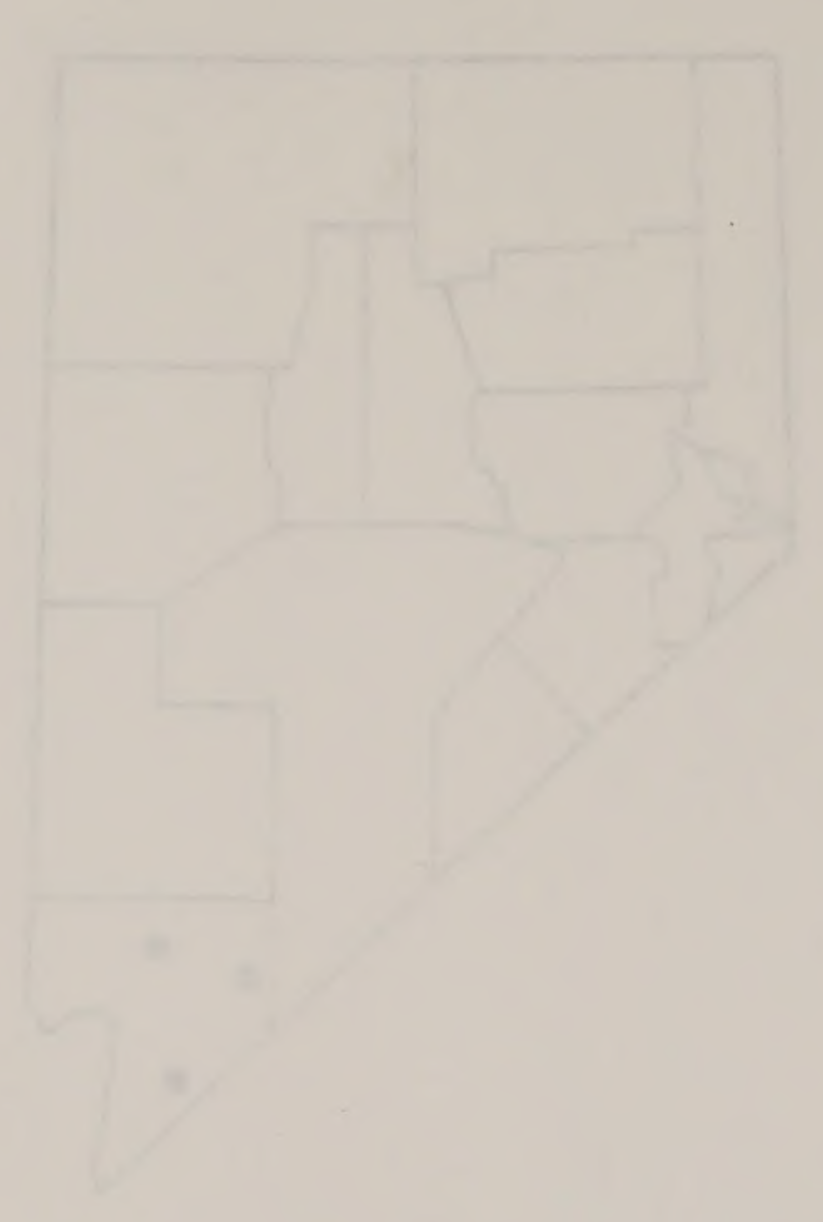

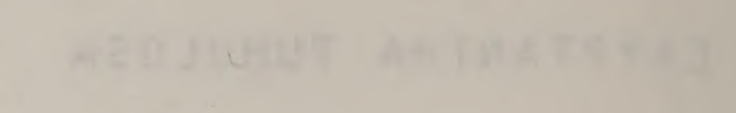

45

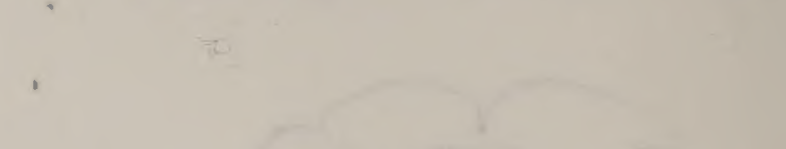


PENSTEMON BICOLOR.

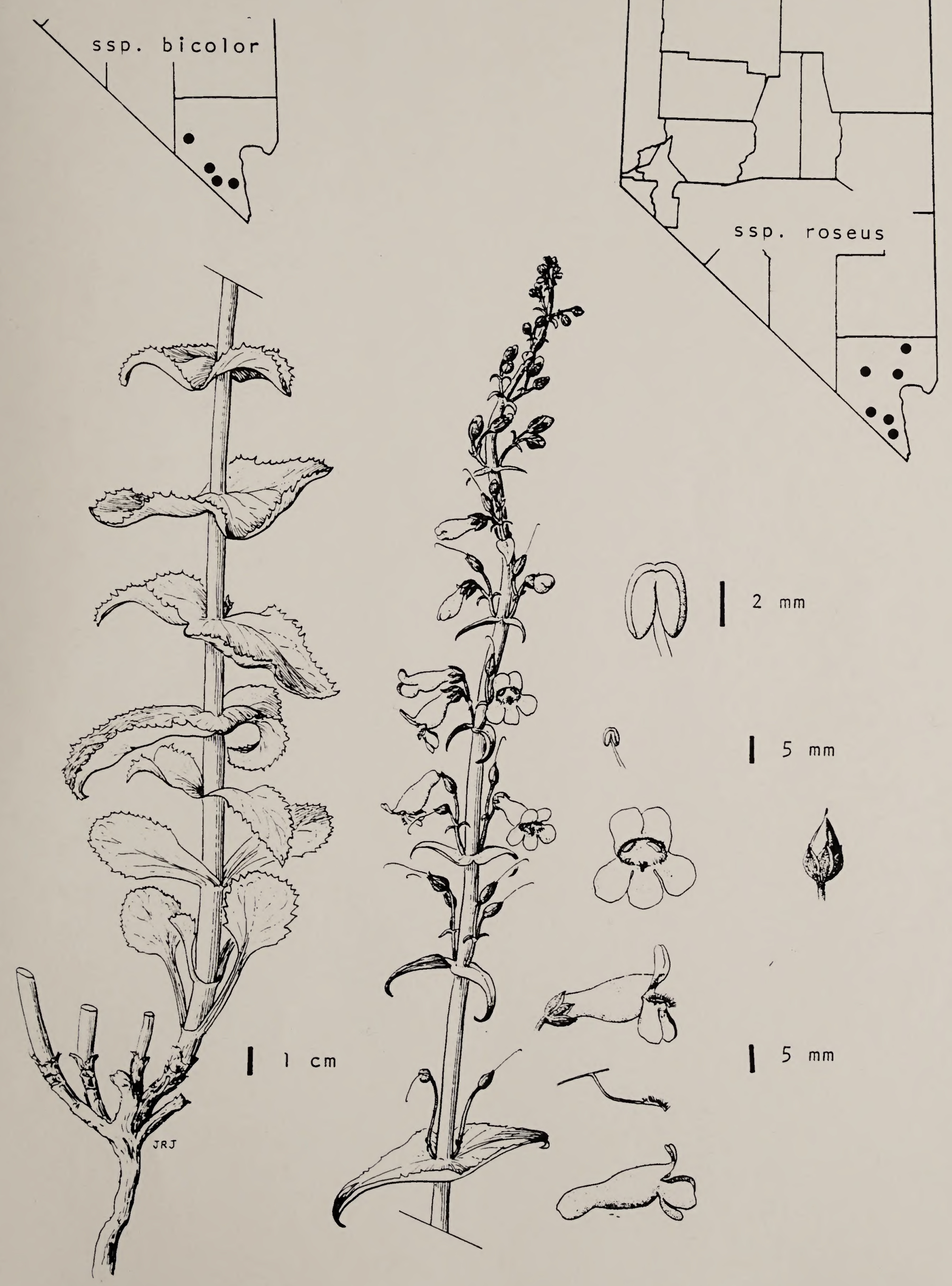




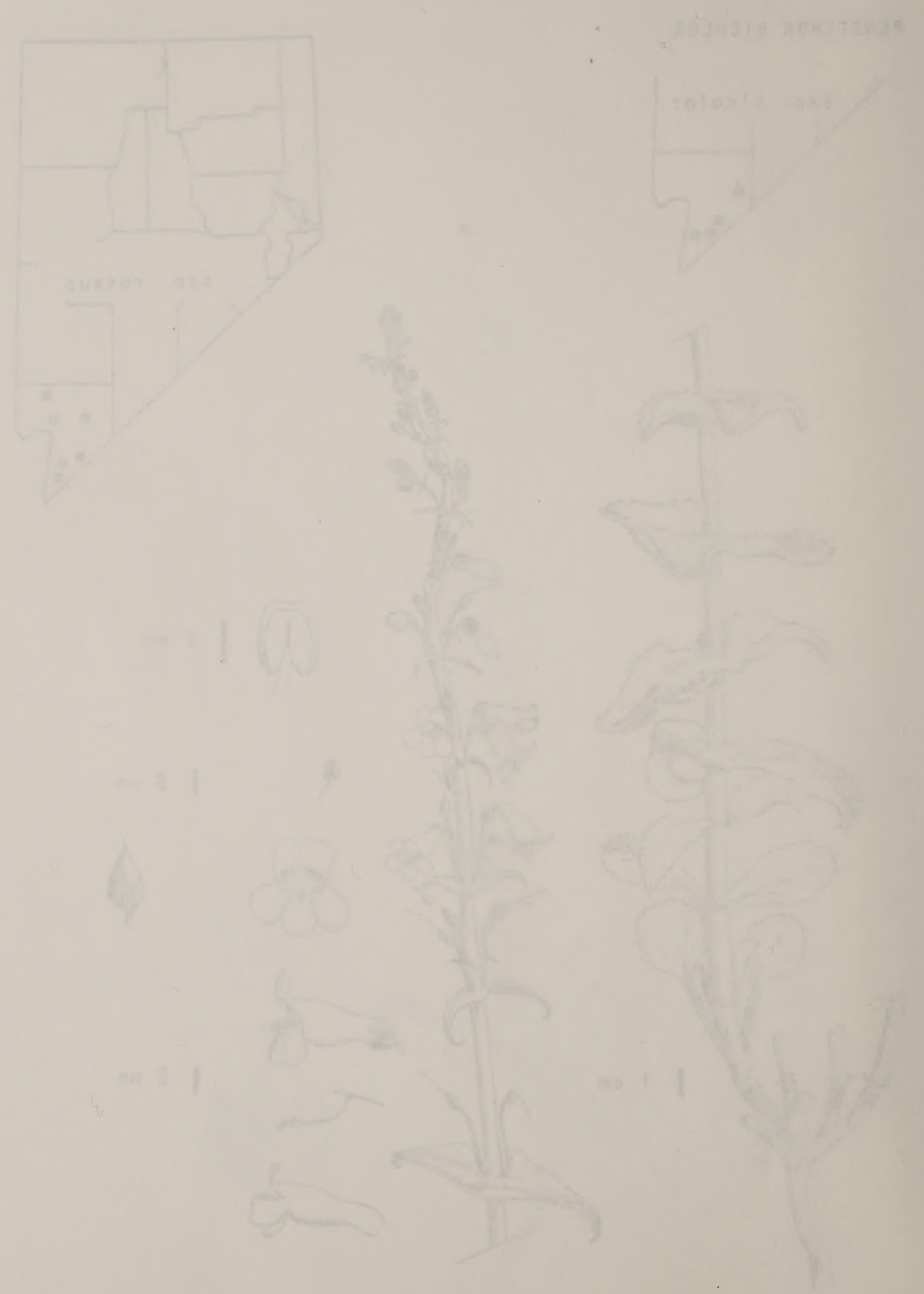



